Supporting Information for

\title{
Computational Study of the Substituent Effects for the Spectroscopic Properties of Thiazolo[5,4-d]thiazole Derivatives
}

\author{
Rongrong Li, ${ }^{a, b}$ Xinzheng Yang ${ }^{*} a, b$
}

\author{
${ }^{a}$ Beijing National Laboratory for Molecular Sciences, State Key Laboratory for Structural \\ Chemistry of Unstable and Stable Species, CAS Research/Education Center for Excellence in \\ Molecular Sciences, Institute of Chemistry Chinese Academy of Sciences, Beijing 100190, P. R. \\ China \\ ${ }^{\mathrm{b}}$ University of Chinese Academy of Sciences, Beijing 100049, P. R. China \\ *E-mail: xyang@iccas.ac.cn
}

\section{Contents}

Table S1. Absolute energies (eV) of the ground states of all molecules .S2-S3

Table S2. Absorption peaks (nm), corresponding oscillator strengths, and primary contributions of synthesized molecules. . .53

Table S3. The molecular orbital compositions of HOMOs and LUMOs....

Table S4. The AIM effective electron numbers of thiazolo[5,4-d]thiazolea $(\rho(\mathrm{I}))$ and two pyridyl $(\rho(\mathrm{II}))$.

Figure S1-S11 Optimized structures with atom labels

.S9-S11

Figure S12-S17 Simulated absorption and emission spectra of $\mathrm{Me}_{2} \mathrm{TTz}^{2+}, \mathrm{Oct}_{2} \mathrm{TTz}^{2+}, \mathrm{Bz}_{2} \mathrm{TTz}^{2+}$, $\mathrm{Met}_{2} \mathrm{TTz}^{2+}, \mathrm{Cbe}_{2} \mathrm{TTz}^{2+}, \mathrm{Trif}_{2} \mathrm{TTz}^{2+}, \mathrm{Cbo}_{2} \mathrm{TTz}^{2+}, \mathrm{Acet}_{2} \mathrm{TTz}^{2+}$ and $\mathrm{Form}_{2} \mathrm{TTz}^{2+}$.... .S12-S14

Figure S18-S23 Simulated vibrationally-resolved absorption and emission spectra of $\mathrm{Met}_{2} \mathrm{TTz}^{2+}$, $\mathrm{Cbe}_{2} \mathrm{TTz}^{2+}, \mathrm{Trif}_{2} \mathrm{TTz}^{2+}, \mathrm{Cbo}_{2} \mathrm{TTz}^{2+}$, Acet $_{2} \mathrm{TTz}^{2+}$ and Form $2 \mathrm{TTz}^{2+}$ ..S15-S17

Figure S24-S32 Frontier molecular orbital diagrams of closed-shell singlet states.

S18-S22 
Table S1. Ground state absolute electronic energies (eV) of all molecules calculated by using the PBE1PBE and M06-2X functionals with the 6-31+G(d,p) basis set.

\begin{tabular}{|c|c|c|c|c|}
\hline Molecule & Functional & $\begin{array}{l}\text { Closed shell } \\
\text { singlet }\end{array}$ & $\begin{array}{l}\text { Open-shell } \\
\text { singlet }\end{array}$ & $\begin{array}{l}\text { Open-shell } \\
\text { triplet }\end{array}$ \\
\hline \multirow[t]{2}{*}{$\mathrm{H}_{2} \mathrm{TTz}^{2+}$} & M06-2X & -42288.752 & -42288.751 & -42286.746 \\
\hline & PBE1PBE & -42267.495 & -42267.495 & -42265.740 \\
\hline \multirow[t]{2}{*}{$\mathrm{Me}_{2} \mathrm{TTz}^{2+}$} & M06-2X & -44425.603 & -44425.603 & -44423.563 \\
\hline & PBE1PBE & -44402.779 & -44402.779 & -44401.039 \\
\hline \multirow[t]{2}{*}{$\mathrm{Oct}_{2} \mathrm{TTz}^{2+}$} & M06-2X & -59385.449 & -59385.449 & -59383.442 \\
\hline & PBE1PBE & -59352.279 & -59352.279 & -59350.735 \\
\hline \multirow[t]{2}{*}{$\mathrm{Bz}_{2} \mathrm{TTz}^{2+}$} & M06-2X & -56991.548 & -56991.529 & -56989.528 \\
\hline & PBE1PBE & -56959.210 & -56959.196 & -56957.432 \\
\hline \multirow[t]{2}{*}{$\mathrm{Met}_{2} \mathrm{TTz}^{2+}$} & M06-2X & -54684.766 & -54684.783 & -54682.787 \\
\hline & PBE1PBE & -54654.955 & -54654.955 & -54653.271 \\
\hline \multirow[t]{2}{*}{$\mathrm{Cbe}_{2} \mathrm{TTz}^{2+}$} & M06-2X & -54680.272 & -54680.293 & -54677.878 \\
\hline & PBE1PBE & -54650.558 & -54650.558 & -54648.854 \\
\hline \multirow[t]{2}{*}{$\operatorname{Trif}_{2} \mathrm{TTz}^{2+}$} & M06-2X & -60624.767 & -60624.767 & -60622.894 \\
\hline & PBE1PBE & -60592.100 & -60592.100 & -60590.488 \\
\hline \multirow[t]{2}{*}{$\mathrm{Eth}_{2} \mathrm{TTz}^{2+}$} & M06-2X & -46496.819 & -46496.819 & -46494.886 \\
\hline & PBE1PBE & -46472.284 & -46472.284 & -46470.661 \\
\hline \multirow[t]{2}{*}{$\mathrm{Cbo}_{2} \mathrm{TTz}^{2+}$} & M06-2X & -52547.013 & -52546.960 & -52545.090 \\
\hline & PBE1PBE & -52518.727 & -52518.728 & -52517.085 \\
\hline \multirow[t]{2}{*}{$\operatorname{Acet}_{2} \mathrm{TTz}^{2+}$} & M06-2X & -50590.830 & -50590.859 & -50588.893 \\
\hline & PBE1PBE & -50563.719 & -50563.719 & -50562.064 \\
\hline \multirow[t]{2}{*}{$\mathrm{Ace}_{2} \mathrm{TTz}^{2+}$} & M06-2X & -46428.874 & -46428.874 & -46426.980 \\
\hline & PBE1PBE & -46403.943 & -46403.943 & -46402.340 \\
\hline Form $_{2} \mathrm{TTz}^{2+}$ & M06-2X & -48452.959 & -48452.933 & -48451.078 \\
\hline
\end{tabular}




\begin{tabular}{rllll} 
& PBE1PBE & -48427.332 & -48427.332 & -48425.735 \\
Cyan $_{2} \mathrm{TTz}^{2+}$ & M06-2X & -47304.592 & -47304.565 & -47302.752 \\
& PBE1PBE & -47279.183 & -47279.183 & -47277.597 \\
\hline
\end{tabular}

Table S2. Absorption peaks (nm), oscillator strengths, and primary molecular orbital contributions of experimentally reported molecules calculated by using the M06-2X functional.

\begin{tabular}{lccc}
\hline Molecule & $\lambda_{\text {abs }} / \mathrm{nm}$ & $f_{\text {os }}(\mathrm{abs})$ & Dominant orbitals \\
\hline $\mathrm{H}_{2} \mathrm{TTz}^{2+}$ & 357.75 & 1.14 & $\mathrm{HOMO} \rightarrow$ LUMO $(97 \%)$ \\
$\mathrm{Me}_{2} \mathrm{TTz}^{2+}$ & 359.99 & 1.30 & $\mathrm{HOMO} \rightarrow$ LUMO $(97 \%)$ \\
$\mathrm{Oct}_{2} \mathrm{TTz}^{2+}$ & 360.58 & 1.44 & $\mathrm{HOMO} \rightarrow$ LUMO $(97 \%)$ \\
$\mathrm{Bz}_{2} \mathrm{TTz}^{2+}$ & 363.77 & 1.47 & $\mathrm{HOMO} \rightarrow$ LUMO $(96 \%)$ \\
\hline
\end{tabular}

Table S3. The contributions of atomic orbitals to the HOMOs and LUMOs of $\mathrm{Me}_{2} \mathrm{TTz}^{2+}$, $\mathrm{Oct}_{2} \mathrm{TTz}^{2+}, \mathrm{Bz}_{2} \mathrm{TTz}^{2+}, \mathrm{Met}_{2} \mathrm{TTz}^{2+}, \mathrm{Cbe}_{2} \mathrm{TTz}^{2+}, \mathrm{Trif}_{2} \mathrm{TTz}^{2+}, \mathrm{Eth}_{2} \mathrm{TTz}^{2+}, \mathrm{Cbo}_{2} \mathrm{TTz}^{2+}, \mathrm{Acet}_{2} \mathrm{TTz}^{2+}$, $\mathrm{Ace}_{2} \mathrm{TTz}^{2+}$, Form $_{2} \mathrm{TTz}^{2+}$, and $\mathrm{Cyan}_{2} \mathrm{TTz}^{2+}$.

\begin{tabular}{|c|c|c|c|c|c|c|c|c|}
\hline \multirow{2}{*}{$\begin{array}{l}\text { Molecule } \\
\mathrm{Me}_{2} \mathrm{TTz}^{2+}\end{array}$} & \multirow{2}{*}{$\begin{array}{l}\text { Orbit } \\
\mathrm{HOMO}\end{array}$} & \multicolumn{7}{|c|}{ The contributions of atomic orbitals } \\
\hline & & $1(\mathrm{C})$ & $18(\mathrm{C})$ & $19(\mathrm{C})$ & $2(\mathrm{C})$ & $4(\mathrm{C})$ & $21(\mathrm{C})$ & $27(\mathrm{C})$ \\
\hline & & $16.38 \%$ & $16.35 \%$ & $15.72 \%$ & $15.72 \%$ & $4.33 \%$ & $4.11 \%$ & $4.07 \%$ \\
\hline & & $10(\mathrm{C})$ & $16(\mathrm{~N})$ & $33(\mathrm{~N})$ & $34(\mathrm{~S})$ & $17(\mathrm{~S})$ & $15(\mathrm{~N})$ & $32(\mathrm{~N})$ \\
\hline & & $3.87 \%$ & $3.80 \%$ & $3.80 \%$ & $2.21 \%$ & $2.21 \%$ & $1.56 \%$ & $1.56 \%$ \\
\hline & LUMO & $2(\mathrm{C})$ & $19(\mathrm{C})$ & $3(\mathrm{C})$ & $20(\mathrm{C})$ & $16(\mathrm{~N})$ & $33(\mathrm{~N})$ & $17(\mathrm{~S})$ \\
\hline & & $8.67 \%$ & $8.67 \%$ & $7.06 \%$ & $7.06 \%$ & $6.52 \%$ & $6.51 \%$ & $5.64 \%$ \\
\hline & & $34(\mathrm{~S})$ & $32(\mathrm{~N})$ & $15(\mathrm{~N})$ & $27(\mathrm{C})$ & $4(\mathrm{C})$ & $23(\mathrm{C})$ & $6(\mathrm{C})$ \\
\hline & & $5.62 \%$ & $4.57 \%$ & $4.57 \%$ & $3.70 \%$ & $3.66 \%$ & $3.63 \%$ & $3.57 \%$ \\
\hline & & $10(\mathrm{C})$ & $21(\mathrm{C})$ & $8(C)$ & $25(\mathrm{C})$ & $18(\mathrm{C})$ & $1(\mathrm{C})$ & \\
\hline & & $3.49 \%$ & $3.43 \%$ & $3.30 \%$ & $3.23 \%$ & $1.74 \%$ & $1.74 \%$ & \\
\hline $\mathrm{Oct}_{2} \mathrm{TTz}^{2+}$ & HOMO & $1(\mathrm{C})$ & $17(\mathrm{C})$ & $2(\mathrm{C})$ & $18(\mathrm{C})$ & $20(C)$ & $4(C)$ & $31(\mathrm{~N})$ \\
\hline
\end{tabular}




\begin{tabular}{|c|c|c|c|c|c|c|c|c|}
\hline & & $16.24 \%$ & $16.24 \%$ & $15.56 \%$ & $15.56 \%$ & $4.57 \%$ & $4.57 \%$ & $3.84 \%$ \\
\hline & & $15(\mathrm{~N})$ & $10(\mathrm{C})$ & $26(C)$ & $16(\mathrm{~S})$ & $32(\mathrm{~S})$ & $30(\mathrm{~N})$ & $14(\mathrm{~N})$ \\
\hline & & $3.84 \%$ & $3.67 \%$ & $3.67 \%$ & $2.18 \%$ & $2.18 \%$ & $1.59 \%$ & $1.59 \%$ \\
\hline & LUMO & $18(\mathrm{C})$ & $2(\mathrm{C})$ & $19(\mathrm{C})$ & $3(\mathrm{C})$ & $31(\mathrm{~N})$ & $15(\mathrm{~N})$ & $16(\mathrm{~S})$ \\
\hline & & $8.79 \%$ & $8.79 \%$ & $6.96 \%$ & $6.96 \%$ & $6.53 \%$ & $6.53 \%$ & $5.66 \%$ \\
\hline & & $32(\mathrm{~S})$ & $14(\mathrm{~N})$ & $30(\mathrm{~N})$ & $20(C)$ & $4(C)$ & $22(\mathrm{C})$ & $6(C)$ \\
\hline & & $5.66 \%$ & $4.59 \%$ & $4.59 \%$ & $3.83 \%$ & $3.83 \%$ & $3.46 \%$ & $3.45 \%$ \\
\hline & & $10(\mathrm{C})$ & $26(C)$ & $8(\mathrm{C})$ & $24(\mathrm{C})$ & $17(\mathrm{C})$ & $1(\mathrm{C})$ & \\
\hline & & $3.38 \%$ & $3.38 \%$ & $3.24 \%$ & $3.24 \%$ & $1.75 \%$ & $1.75 \%$ & \\
\hline $\mathrm{Bz}_{2} \mathrm{TTz}^{2+}$ & HOMO & $1(\mathrm{C})$ & $29(C)$ & $30(\mathrm{C})$ & $2(\mathrm{C})$ & $32(\mathrm{C})$ & $4(C)$ & $55(\mathrm{~N})$ \\
\hline & & $15.20 \%$ & $15.20 \%$ & $14.61 \%$ & $14.61 \%$ & $3.94 \%$ & $3.93 \%$ & $3.77 \%$ \\
\hline & & $38(\mathrm{C})$ & $10(\mathrm{C})$ & $27(\mathrm{~N})$ & $56(\mathrm{~S})$ & $28(\mathrm{~S})$ & $54(\mathrm{~N})$ & $26(\mathrm{~N})$ \\
\hline & & $3.77 \%$ & $3.77 \%$ & $3.77 \%$ & $2.06 \%$ & $2.06 \%$ & $1.46 \%$ & $1.46 \%$ \\
\hline & & $43(\mathrm{C})$ & $15(\mathrm{C})$ & $48(\mathrm{C})$ & $20(\mathrm{C})$ & & & \\
\hline & & $1.31 \%$ & $1.31 \%$ & $1.13 \%$ & $1.13 \%$ & & & \\
\hline & LUMO & $30(\mathrm{C})$ & $2(\mathrm{C})$ & $31(\mathrm{C})$ & $3(\mathrm{C})$ & $55(\mathrm{~N})$ & $27(\mathrm{~N})$ & $56(\mathrm{~S})$ \\
\hline & & $8.54 \%$ & $8.54 \%$ & $7.15 \%$ & $7.14 \%$ & $6.44 \%$ & $6.44 \%$ & $5.59 \%$ \\
\hline & & $28(\mathrm{~S})$ & $54(\mathrm{~N})$ & $26(\mathrm{~N})$ & $34(\mathrm{C})$ & $6(C)$ & $10(\mathrm{C})$ & $38(\mathrm{C})$ \\
\hline & & $5.58 \%$ & $4.56 \%$ & $4.55 \%$ & $3.70 \%$ & $3.69 \%$ & $3.60 \%$ & $3.60 \%$ \\
\hline & & $32(\mathrm{C})$ & $4(\mathrm{C})$ & $36(\mathrm{C})$ & $8(\mathrm{C})$ & $29(\mathrm{C})$ & $1(\mathrm{C})$ & \\
\hline & & $3.45 \%$ & $3.45 \%$ & $3.35 \%$ & $3.35 \%$ & $1.72 \%$ & $1.71 \%$ & \\
\hline $\mathrm{Met}_{2} \mathrm{TTz}^{2+}$ & HOMO & $1(\mathrm{C})$ & $14(\mathrm{C})$ & $15(\mathrm{C})$ & $2(\mathrm{C})$ & $17(\mathrm{C})$ & $4(\mathrm{C})$ & $23(\mathrm{C})$ \\
\hline & & $16.22 \%$ & $16.22 \%$ & $15.80 \%$ & $15.80 \%$ & $4.13 \%$ & $4.13 \%$ & $4.01 \%$ \\
\hline & & $10(\mathrm{C})$ & $34(\mathrm{~N})$ & $33(\mathrm{~N})$ & $13(\mathrm{~S})$ & $26(\mathrm{~S})$ & $25(\mathrm{~N})$ & $12(\mathrm{~N})$ \\
\hline & & $4.01 \%$ & $3.69 \%$ & $3.69 \%$ & $2.16 \%$ & $2.16 \%$ & $1.45 \%$ & $1.45 \%$ \\
\hline & LUMO & $15(\mathrm{C})$ & $2(\mathrm{C})$ & $16(\mathrm{C})$ & $3(\mathrm{C})$ & $34(\mathrm{~N})$ & $33(\mathrm{~N})$ & $13(\mathrm{~S})$ \\
\hline & & $8.14 \%$ & $8.14 \%$ & $7.52 \%$ & $7.52 \%$ & $6.37 \%$ & $6.36 \%$ & $5.50 \%$ \\
\hline
\end{tabular}


$26(\mathrm{~S}) \quad 25(\mathrm{~N}) \quad 12(\mathrm{~N}) \quad 19(\mathrm{C}) \quad 6(\mathrm{C}) \quad 21(\mathrm{C}) \quad 8(\mathrm{C})$

$5.50 \% \quad 4.53 \% \quad 4.53 \% \quad 4.05 \% \quad 4.05 \% \quad 3.69 \% \quad 3.69 \%$

$10(\mathrm{C}) \quad 23(\mathrm{C}) \quad 17(\mathrm{C}) \quad 4(\mathrm{C}) \quad 14(\mathrm{C}) \quad 1(\mathrm{C})$

$3.48 \% \quad 3.47 \% \quad 3.32 \% \quad 3.32 \% \quad 1.63 \% \quad 1.63 \%$

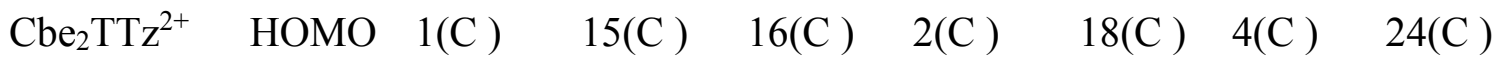

$16.44 \% \quad 16.40 \% \quad 16.16 \% \quad 16.16 \% \quad 4.08 \% \quad 4.04 \% \quad 3.99 \%$

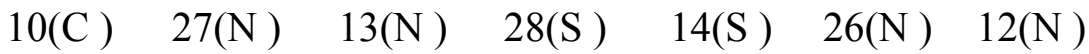

$3.96 \% \quad 3.45 \% \quad 3.41 \% \quad 2.19 \% \quad 2.18 \% \quad 1.35 \% \quad 1.35 \%$

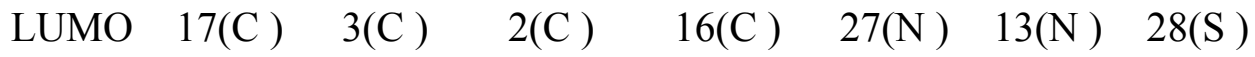

$\begin{array}{lllllll}7.99 \% & 7.89 \% & 7.58 \% & 7.57 \% & 6.36 \% & 6.30 \% & 5.37 \%\end{array}$

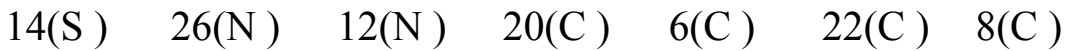

$5.30 \% \quad 4.48 \% \quad 4.41 \% \quad 4.40 \% \quad 4.34 \% \quad 4.03 \% \quad 4.01 \%$

24(C) $\quad 10(\mathrm{C}) \quad 18(\mathrm{C}) \quad 4(\mathrm{C}) \quad 15(\mathrm{C}) \quad 1(\mathrm{C})$

$3.38 \% \quad 3.36 \% \quad 3.23 \% \quad 3.20 \% \quad 1.53 \% \quad 1.49 \%$

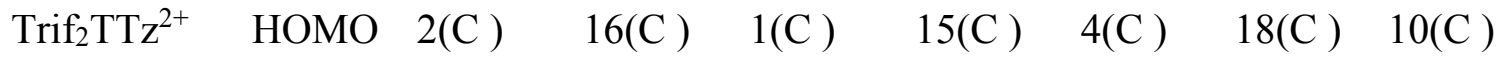

$\begin{array}{lllllll}16.87 \% & 16.87 \% & 16.73 \% & 16.73 \% & 3.99 \% & 3.99 \% & 3.93 \%\end{array}$

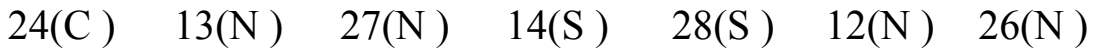

$3.93 \% \quad 3.12 \% \quad 3.12 \% \quad 2.20 \% \quad 2.20 \% \quad 1.15 \% \quad 1.15 \%$

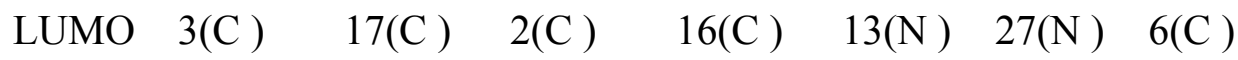

$\begin{array}{lllllll}8.83 \% & 8.83 \% & 6.71 \% & 6.71 \% & 5.91 \% & 5.91 \% & 5.25 \%\end{array}$

$20(\mathrm{C}) \quad 14(\mathrm{~S}) \quad 28(\mathrm{~S}) \quad 8(\mathrm{C}) \quad 22(\mathrm{C}) \quad 12(\mathrm{~N}) \quad 26(\mathrm{~N})$

$5.25 \% \quad 5.15 \% \quad 5.15 \% \quad 4.93 \% \quad 4.93 \% \quad 4.36 \% \quad 4.36 \%$

$10(\mathrm{C}) \quad 24(\mathrm{C}) \quad 4(\mathrm{C}) \quad 18(\mathrm{C}) \quad 1(\mathrm{C}) \quad 15(\mathrm{C})$

$2.93 \% \quad 2.93 \% \quad 2.80 \% \quad 2.80 \% \quad 1.32 \% \quad 1.32 \%$

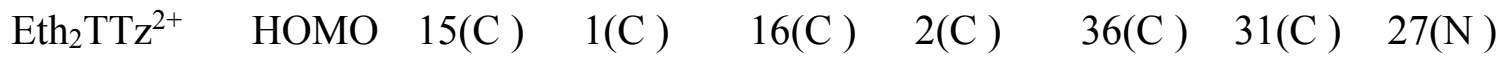

$14.05 \% \quad 14.05 \% \quad 13.47 \% \quad 13.46 \% \quad 4.49 \% \quad 4.39 \% \quad 4.02 \%$

$13(\mathrm{~N}) \quad 18(\mathrm{C}) \quad 4(\mathrm{C}) \quad 24(\mathrm{C}) \quad 10(\mathrm{C}) \quad 34(\mathrm{C}) \quad 29(\mathrm{C})$ 


\begin{tabular}{|c|c|c|c|c|c|c|c|c|}
\hline & & $3.96 \%$ & $3.85 \%$ & $3.79 \%$ & $3.63 \%$ & $3.57 \%$ & $1.91 \%$ & $1.85 \%$ \\
\hline & & $28(\mathrm{~S})$ & $14(\mathrm{~S})$ & $26(\mathrm{~N})$ & $12(\mathrm{~N})$ & & & \\
\hline & & $1.83 \%$ & $1.80 \%$ & $1.55 \%$ & $1.53 \%$ & & & \\
\hline & LUMO & $3(\mathrm{C})$ & $17(\mathrm{C})$ & $16(\mathrm{C})$ & $2(\mathrm{C})$ & $27(\mathrm{~N})$ & $13(\mathrm{~N})$ & $28(\mathrm{~S})$ \\
\hline & & $7.46 \%$ & $7.45 \%$ & $7.31 \%$ & $7.30 \%$ & $5.95 \%$ & $5.95 \%$ & $5.08 \%$ \\
\hline & & $14(\mathrm{~S})$ & $26(\mathrm{~N})$ & $12(\mathrm{~N})$ & $6(C)$ & $20(C)$ & $8(C)$ & $22(\mathrm{C})$ \\
\hline & & $5.07 \%$ & $4.25 \%$ & $4.21 \%$ & $4.10 \%$ & $4.08 \%$ & $3.96 \%$ & $3.93 \%$ \\
\hline & & $31(\mathrm{C})$ & $36(C)$ & $18(\mathrm{C})$ & $4(C)$ & $24(C)$ & $10(\mathrm{C})$ & $15(\mathrm{C})$ \\
\hline & & $3.21 \%$ & $3.18 \%$ & $3.15 \%$ & $3.15 \%$ & $2.95 \%$ & $2.92 \%$ & $1.45 \%$ \\
\hline & & $1(\mathrm{C})$ & & & & & & \\
\hline & & $1.43 \%$ & & & & & & \\
\hline $\mathrm{Cbo}_{2} \mathrm{TTz}^{2+}$ & HOMO & $16(\mathrm{C})$ & $2(\mathrm{C})$ & $1(\mathrm{C})$ & $15(\mathrm{C})$ & $4(C)$ & $18(\mathrm{C})$ & $10(\mathrm{C})$ \\
\hline & & $16.60 \%$ & $16.59 \%$ & $16.43 \%$ & $16.43 \%$ & $4.09 \%$ & $4.09 \%$ & $3.90 \%$ \\
\hline & & $24(\mathrm{C})$ & $13(\mathrm{~N})$ & $27(\mathrm{~N})$ & $14(\mathrm{~S})$ & $28(\mathrm{~S})$ & $12(\mathrm{~N})$ & $26(\mathrm{~N})$ \\
\hline & & $3.90 \%$ & $3.18 \%$ & $3.18 \%$ & $2.12 \%$ & $2.12 \%$ & $1.19 \%$ & $1.19 \%$ \\
\hline & LUMO & $3(\mathrm{C})$ & $17(\mathrm{C})$ & $2(\mathrm{C})$ & $16(\mathrm{C})$ & $6(\mathrm{C})$ & $20(C)$ & $8(C)$ \\
\hline & & $8.68 \%$ & $8.68 \%$ & $6.06 \%$ & $6.06 \%$ & $5.45 \%$ & $5.45 \%$ & $5.17 \%$ \\
\hline & & $22(\mathrm{C})$ & $13(\mathrm{~N})$ & $27(\mathrm{~N})$ & $14(\mathrm{~S})$ & $28(\mathrm{~S})$ & $12(\mathrm{~N})$ & $26(\mathrm{~N})$ \\
\hline & & $5.17 \%$ & $5.08 \%$ & $5.08 \%$ & $4.79 \%$ & $4.79 \%$ & $4.11 \%$ & $4.11 \%$ \\
\hline & & $10(\mathrm{C})$ & $24(\mathrm{C})$ & $4(C)$ & $18(\mathrm{C})$ & $31(\mathrm{O})$ & $32(\mathrm{O})$ & $29(\mathrm{C})$ \\
\hline & & $2.54 \%$ & $2.54 \%$ & $2.46 \%$ & $2.46 \%$ & $1.52 \%$ & $1.52 \%$ & $1.44 \%$ \\
\hline & & $30(\mathrm{C})$ & $1(\mathrm{C})$ & $15(\mathrm{C})$ & & & & \\
\hline & & $1.44 \%$ & $1.20 \%$ & $1.20 \%$ & & & & \\
\hline $\operatorname{Acet}_{2} \mathrm{TTz}^{2+}$ & HOMO & $2(\mathrm{C})$ & $16(\mathrm{C})$ & $1(\mathrm{C})$ & $15(\mathrm{C})$ & $4(C)$ & $18(\mathrm{C})$ & $10(\mathrm{C})$ \\
\hline & & $16.45 \%$ & $16.45 \%$ & $16.39 \%$ & $16.39 \%$ & $4.19 \%$ & $4.19 \%$ & $3.84 \%$ \\
\hline & & $24(C)$ & $13(\mathrm{~N})$ & $27(\mathrm{~N})$ & $14(\mathrm{~S})$ & $28(\mathrm{~S})$ & $12(\mathrm{~N})$ & $26(\mathrm{~N})$ \\
\hline & & $3.84 \%$ & $3.25 \%$ & $3.25 \%$ & $2.13 \%$ & $2.13 \%$ & $1.24 \%$ & $1.24 \%$ \\
\hline
\end{tabular}




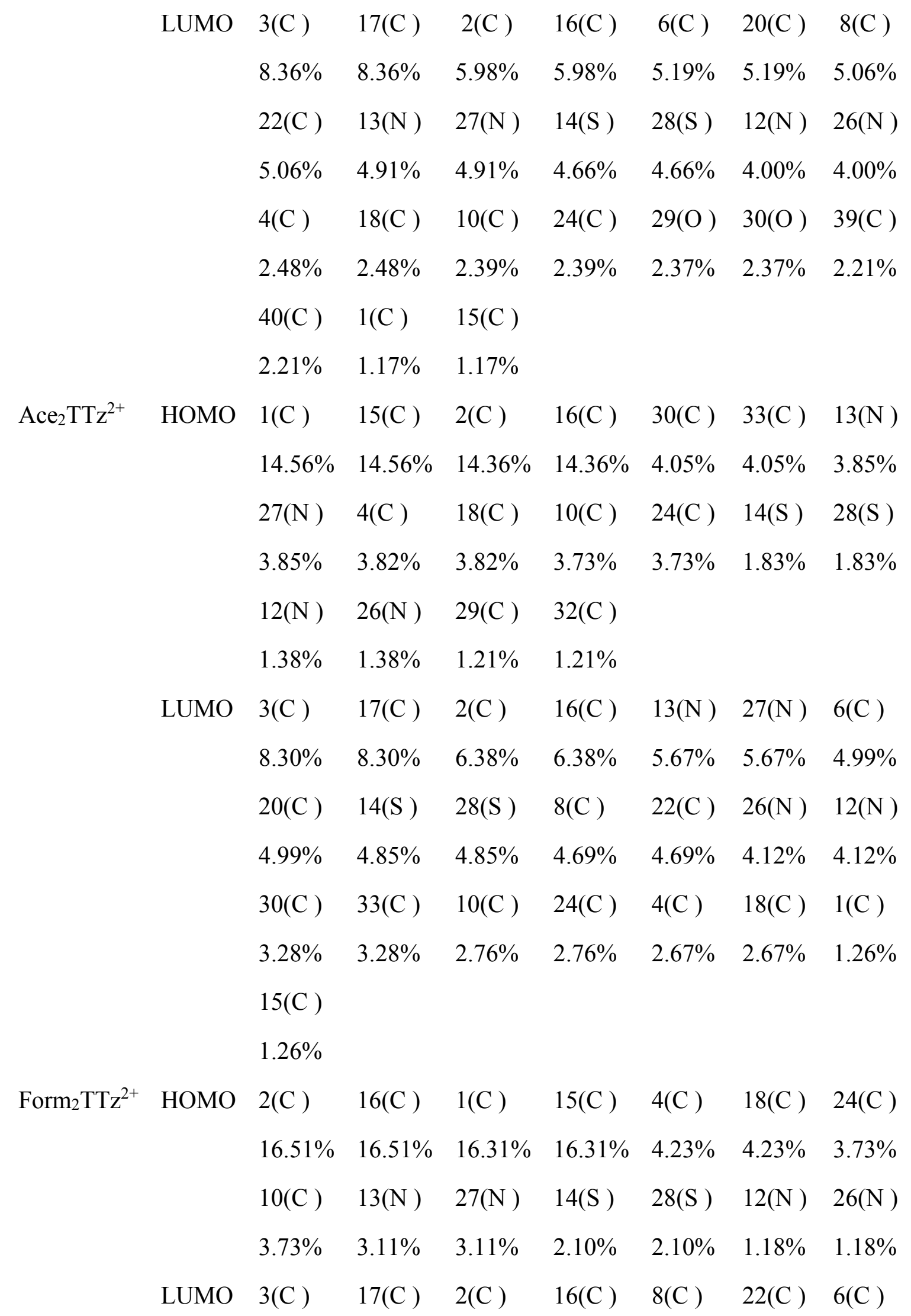




$\begin{array}{lllllll}8.63 \% & 8.63 \% & 5.53 \% & 5.53 \% & 5.41 \% & 5.41 \% & 5.32 \% \\ 20(\mathrm{C}) & 14(\mathrm{~S}) & 28(\mathrm{~S}) & 13(\mathrm{~N}) & 27(\mathrm{~N}) & 12(\mathrm{~N}) & 26(\mathrm{~N}) \\ 5.32 \% & 4.52 \% & 4.52 \% & 4.40 \% & 4.40 \% & 3.90 \% & 3.90 \% \\ 31(\mathrm{O}) & 34(\mathrm{O}) & 29(\mathrm{C}) & 32(\mathrm{C}) & 4(\mathrm{C}) & 18(\mathrm{C}) & 10(\mathrm{C}) \\ 2.91 \% & 2.91 \% & 2.87 \% & 2.87 \% & 2.53 \% & 2.53 \% & 2.00 \% \\ 24(\mathrm{C}) & 1(\mathrm{C}) & 15(\mathrm{C}) & & & & \\ 2.00 \% & 1.07 \% & 1.07 \% & & & & \end{array}$

Table S4. The AIM effective electrons of thiazolo[5,4-d]thiazolea $(\rho(\mathrm{I}))$ and two pyridyl $(\rho(\mathrm{II}))$ in $\mathrm{Oct}_{2} \mathrm{TTz}^{2+}, \mathrm{Bz}_{2} \mathrm{TTz}^{2+}, \mathrm{Met}_{2} \mathrm{TTz}^{2+}, \mathrm{Cbe}_{2} \mathrm{TTz}^{2+}, \mathrm{Trif}_{2} \mathrm{TTz}^{2+}, \mathrm{Eth}_{2} \mathrm{TTz}^{2+}, \mathrm{Cbo}_{2} \mathrm{TTz}^{2+}, \mathrm{Acet}_{2} \mathrm{TTz}^{2+}$, and $\mathrm{Ace}_{2} \mathrm{TTz}^{2+}$.

\begin{tabular}{lll}
\hline Molecule & $\rho(\mathrm{I})$ & $\rho(\mathrm{II})$ \\
\hline $\mathrm{Oct}_{2} \mathrm{TTz}^{2+}$ & 17.58 & 97.48 \\
$\mathrm{Bz}_{2} \mathrm{TTz}^{2+}$ & 27.21 & 118.76 \\
$\mathrm{Met}_{2} \mathrm{TTz}^{2+}$ & 46.66 & 106.50 \\
$\mathrm{Cbe}_{2} \mathrm{TTz}^{2+}$ & 41.55 & 111.70 \\
$\mathrm{Trif}_{2} \mathrm{TTz}^{2+}$ & 65.06 & 116.91 \\
$\mathrm{Eth}_{2} \mathrm{TTz}^{2+}$ & 36.88 & 124.67 \\
$\mathrm{Cbo}_{2} \mathrm{TTz}^{2+}$ & 41.95 & 134.95 \\
$\mathrm{Acet}_{2} \mathrm{TTz}^{2+}$ & 37.50 & 118.38 \\
$\mathrm{Ace}_{2} \mathrm{TTz}^{2+}$ & 58.82 & 112.27 \\
\hline
\end{tabular}




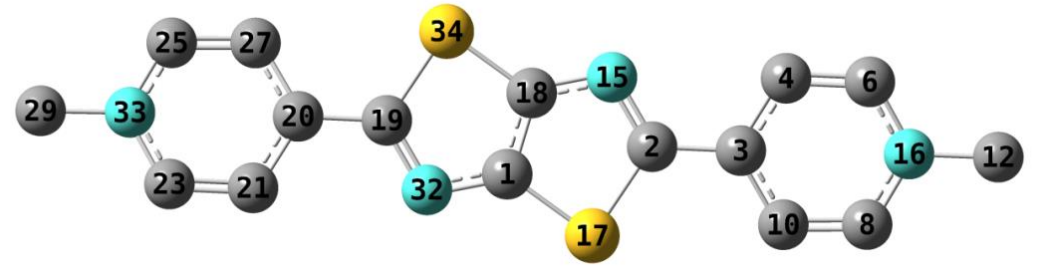

Figure S1. Optimized ground state structure of $\mathrm{Me}_{2} \mathrm{TTz}^{2+}$ with atom labels.

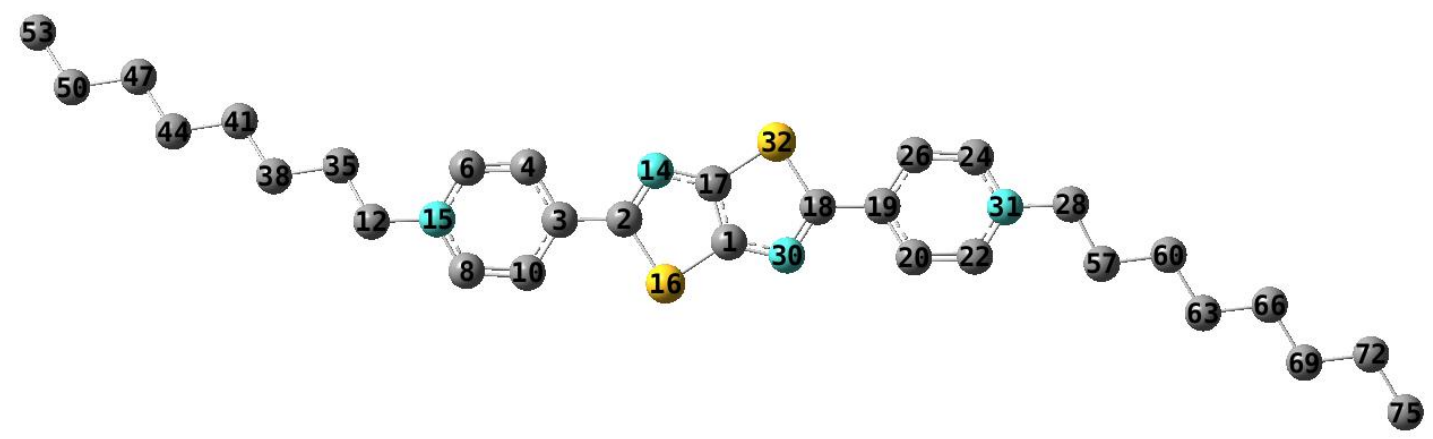

Figure S2. Optimized ground state structure of $\mathrm{Oct}_{2} \mathrm{TTz}^{2+}$ with atom labels.

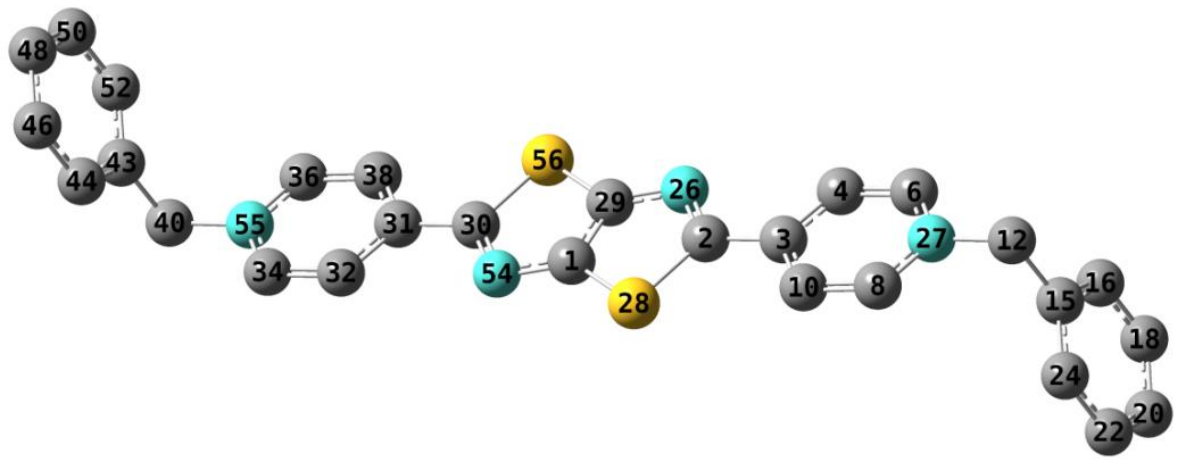

Figure S3. Optimized ground state structure of $\mathrm{Bz}_{2} \mathrm{TTz}^{2+}$ with atom labels. 


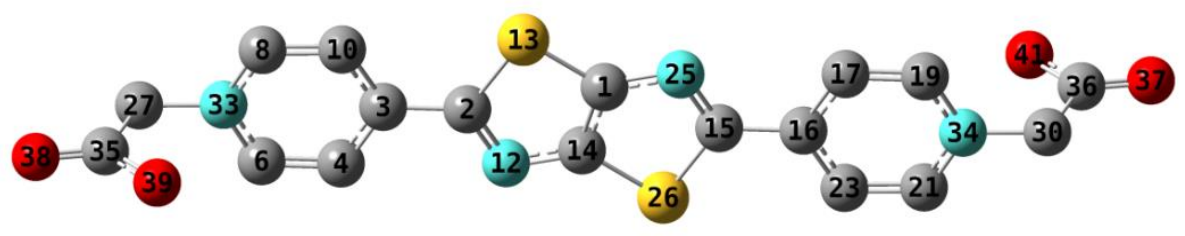

Figure S4. Optimized ground state structure of $\mathrm{Met}_{2} \mathrm{TTz}^{2+}$ with atom labels.

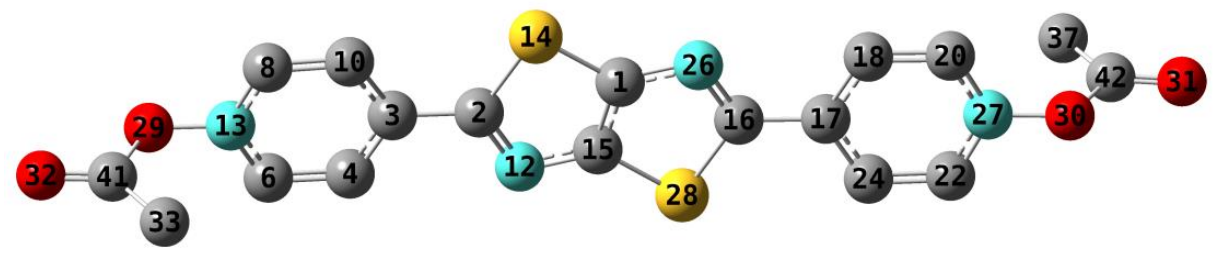

Figure S5. Optimized ground state structure of $\mathrm{Cbe}_{2} \mathrm{TTz}^{2+}$ with atom labels.

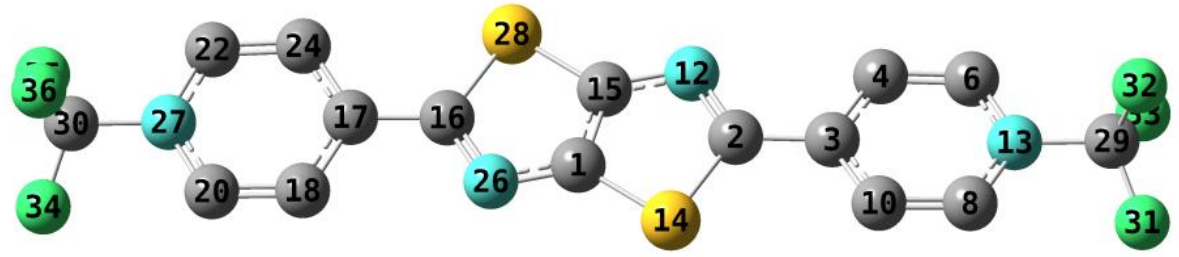

Figure S6. Optimized ground state structure of $\mathrm{Trif}_{2} \mathrm{TTz}^{2+}$ with atom labels.

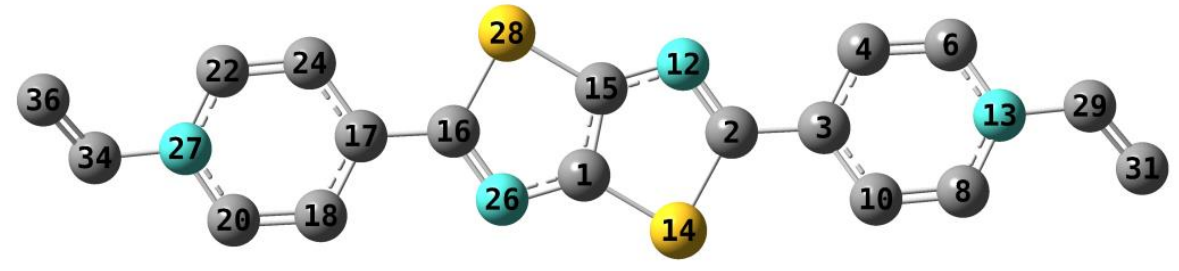

Figure S7. Optimized ground state structure of $\mathrm{Eth}_{2} \mathrm{TTz}^{2+}$ with atom labels. 


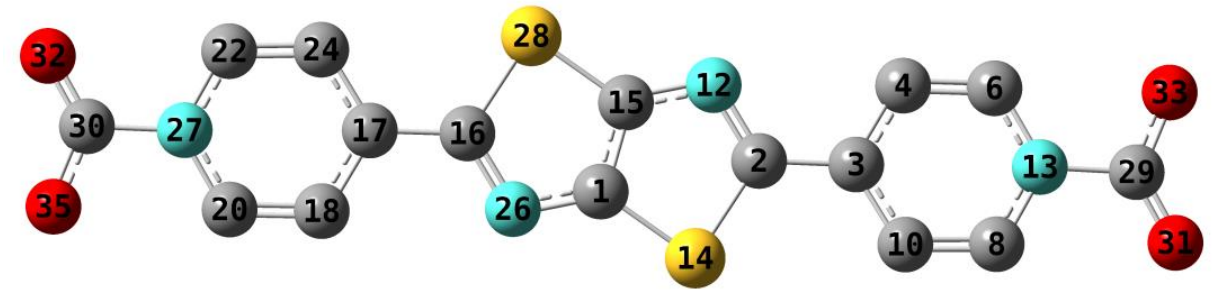

Figure S8. Optimized ground state structure of $\mathrm{Cbo}_{2} \mathrm{TTz}^{2+}$ with atom labels.

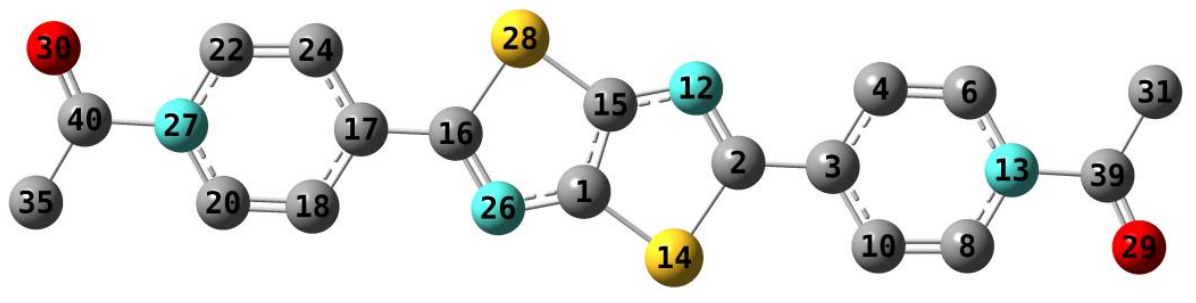

Figure S9. Optimized ground state structure of $\mathrm{Acet}_{2} \mathrm{TTz}^{2+}$ with atom labels.

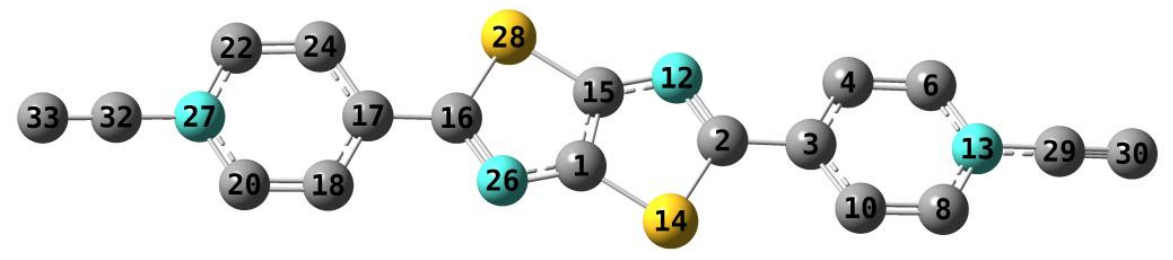

Figure S10. Optimized ground state structure of $\mathrm{Ace}_{2} \mathrm{TTz}^{2+}$ with atom labels.

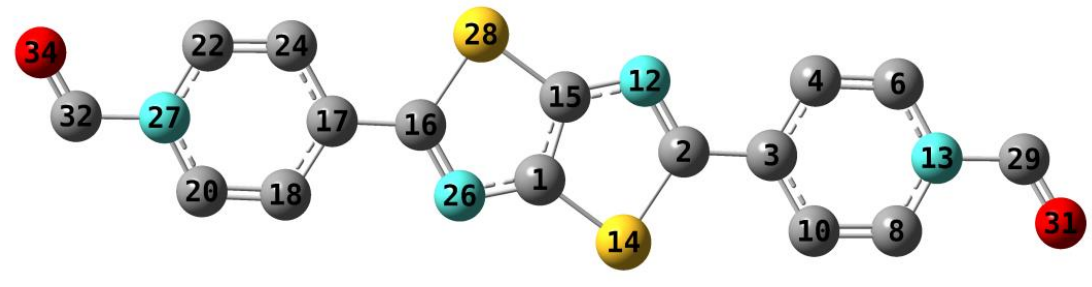

Figure S11. Optimized ground state structure of Form $2 \mathrm{TTz}^{2+}$ with atom labels. 


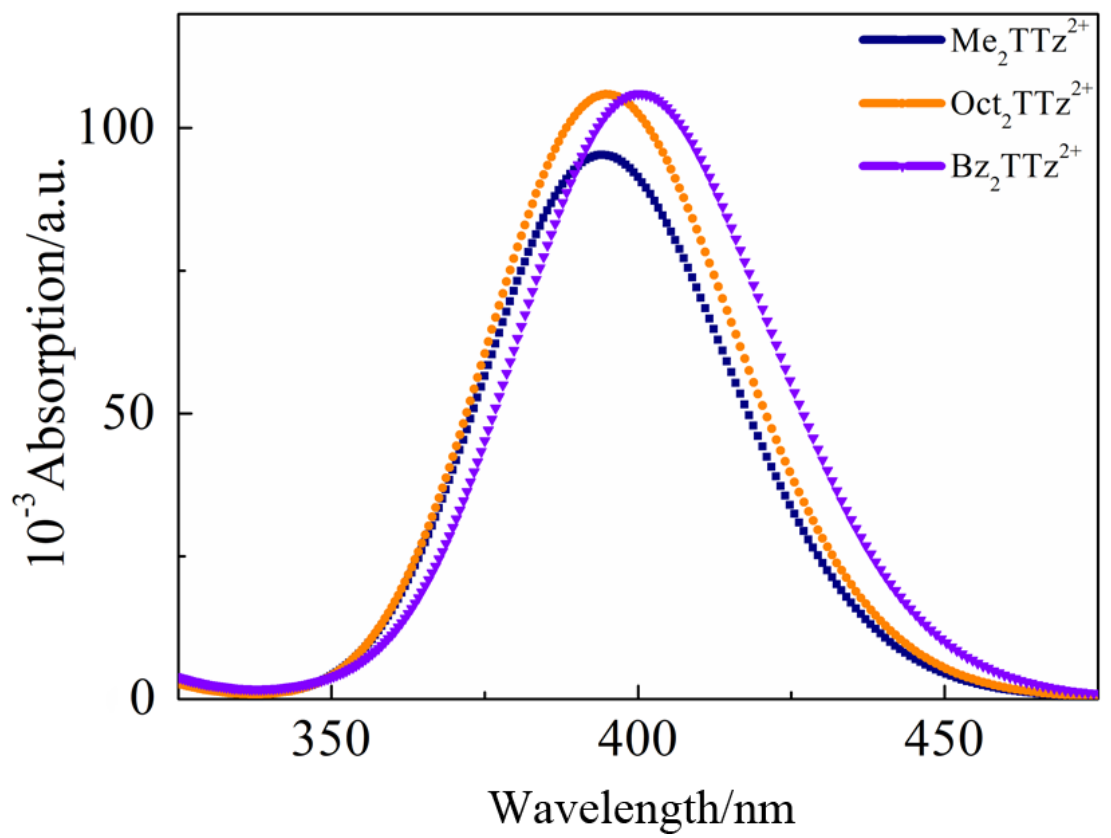

Figure S12. Simulated absorption spectra of $\mathrm{Me}_{2} \mathrm{TTz}^{2+}, \mathrm{Oct}_{2} \mathrm{TTz}^{2+}$ and $\mathrm{Bz}_{2} \mathrm{TTz}^{2+}$ at the PBE1PBE/6-31+G(d,p) level.

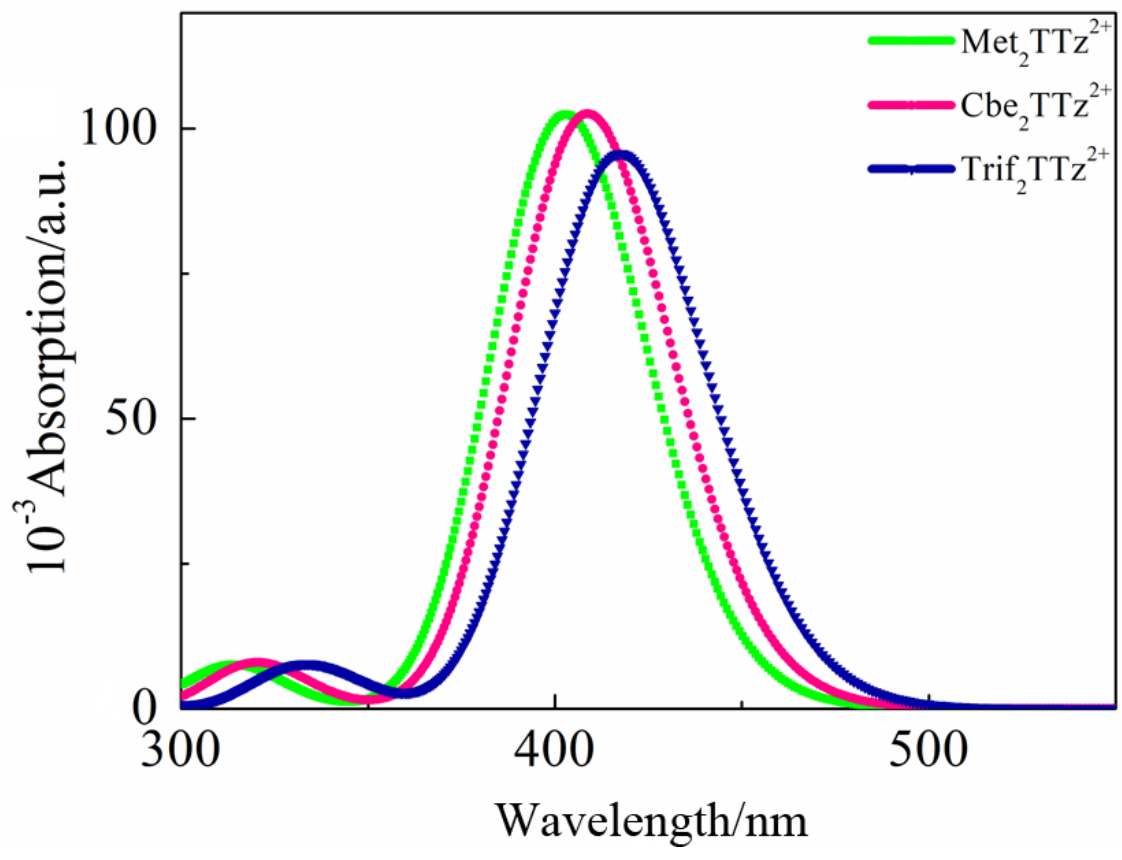

Figure S13. Simulated absorption spectra of $\mathrm{Met}_{2} \mathrm{TTz}^{2+}, \mathrm{Cbe}_{2} \mathrm{TTz}^{2+}$ and $\mathrm{Trif}_{2} \mathrm{TTz}^{2+}$ at the PBE1PBE/6-31+G(d,p) level. 


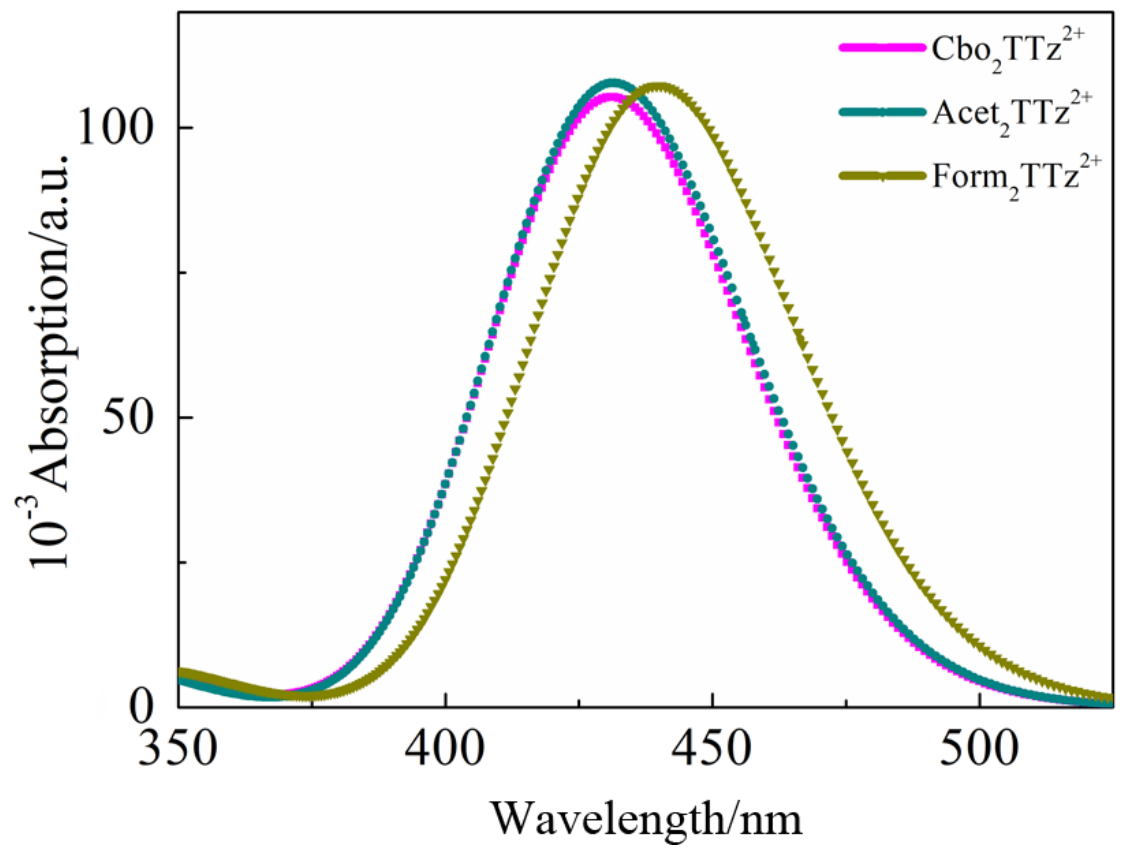

Figure S14. Simulated absorption spectra of $\mathrm{Cbo}_{2} \mathrm{TTz}^{2+}, \mathrm{Acet}_{2} \mathrm{TTz}^{2+}$ and $\mathrm{Form}_{2} \mathrm{TTz}^{2+}$ at the PBE1PBE/6-31+G(d,p) level.

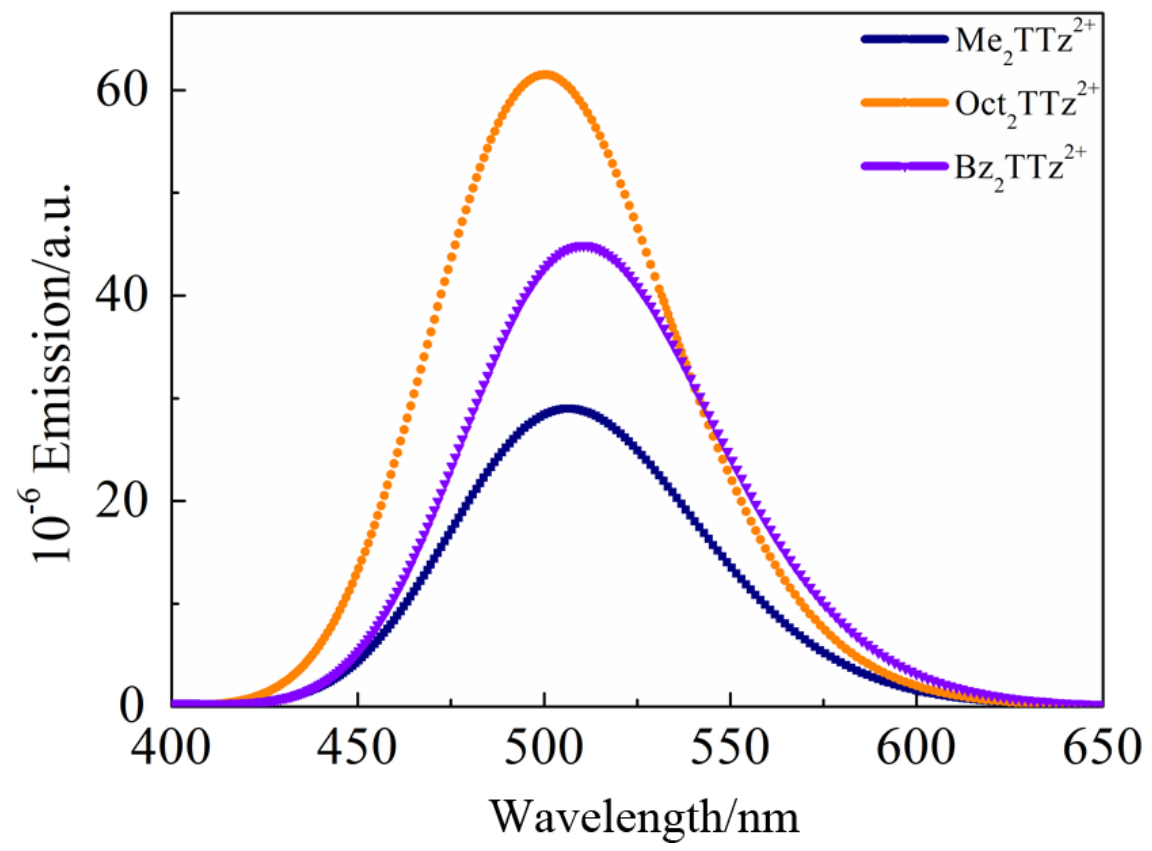

Figure S15. Simulated emission spectra of $\mathrm{Me}_{2} \mathrm{TTz}^{2+}$, $\mathrm{Oct}_{2} \mathrm{TTz}^{2+}$ and $\mathrm{Bz}_{2} \mathrm{TTz}^{2+}$ at the PBE1PBE/6-31+G(d,p) level. 


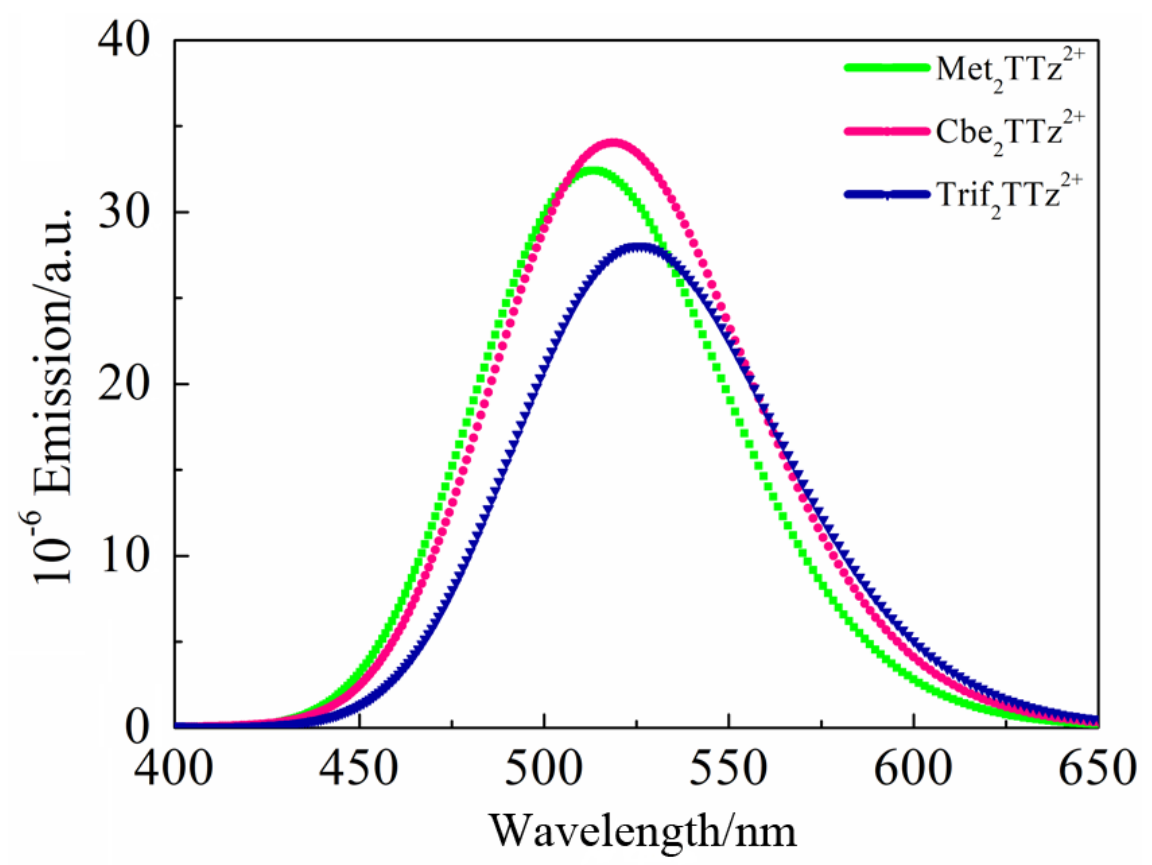

Figure S16. Simulated emission spectra of $\mathrm{Met}_{2} \mathrm{TTz}^{2+}, \mathrm{Cbe}_{2} \mathrm{TTz}^{2+}$ and $\mathrm{Trif}_{2} \mathrm{TTz}^{2+}$ at the PBE1PBE/6-31+G(d,p) level.

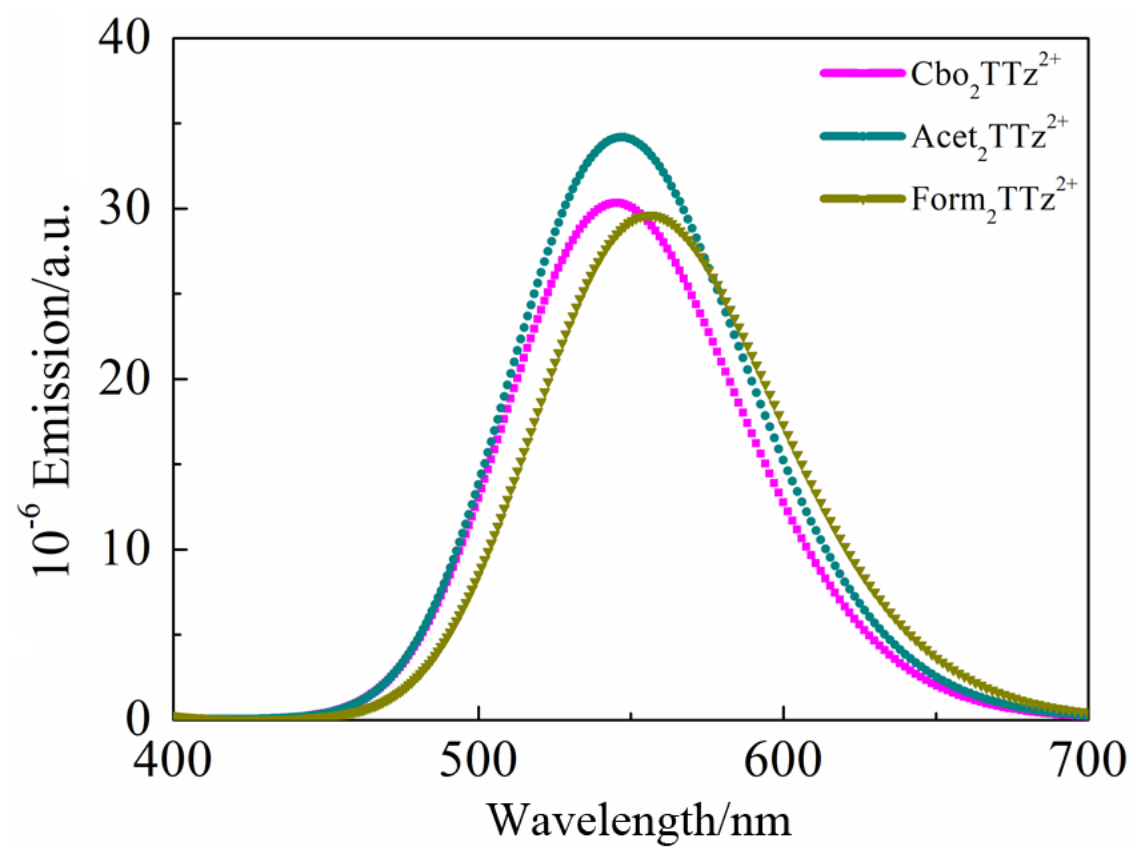

Figure S17. Simulated emission spectra of $\mathrm{Cbo}_{2} \mathrm{TTz}^{2+}$, $\mathrm{Acet}_{2} \mathrm{TTz}^{2+}$ and $\mathrm{Form}_{2} \mathrm{TTz}^{2+}$ at the PBE1PBE/6-31+G(d,p) level. 


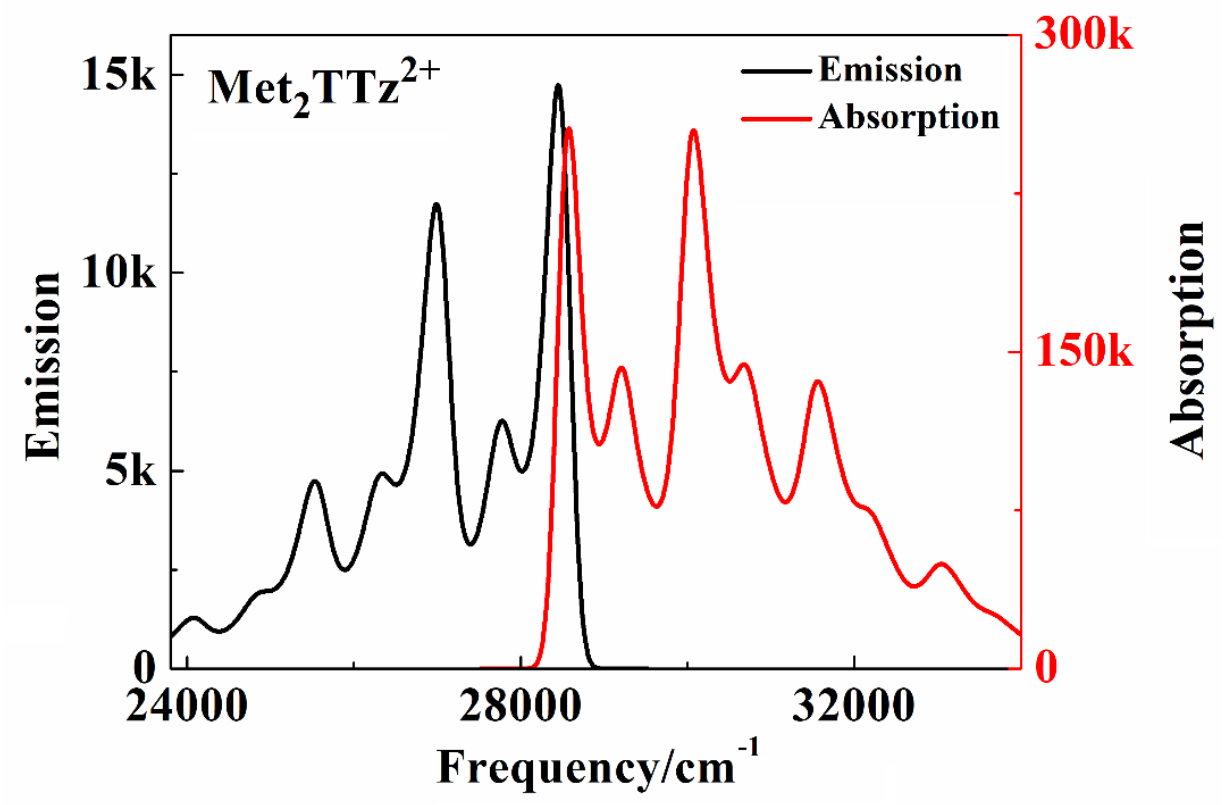

Figure S18. Vibrationally-resolved absorption and emission spectra of $\mathrm{Met}_{2} \mathrm{TTz}^{2+}$ at the PBE1PBE/6-31+G(d,p) level.

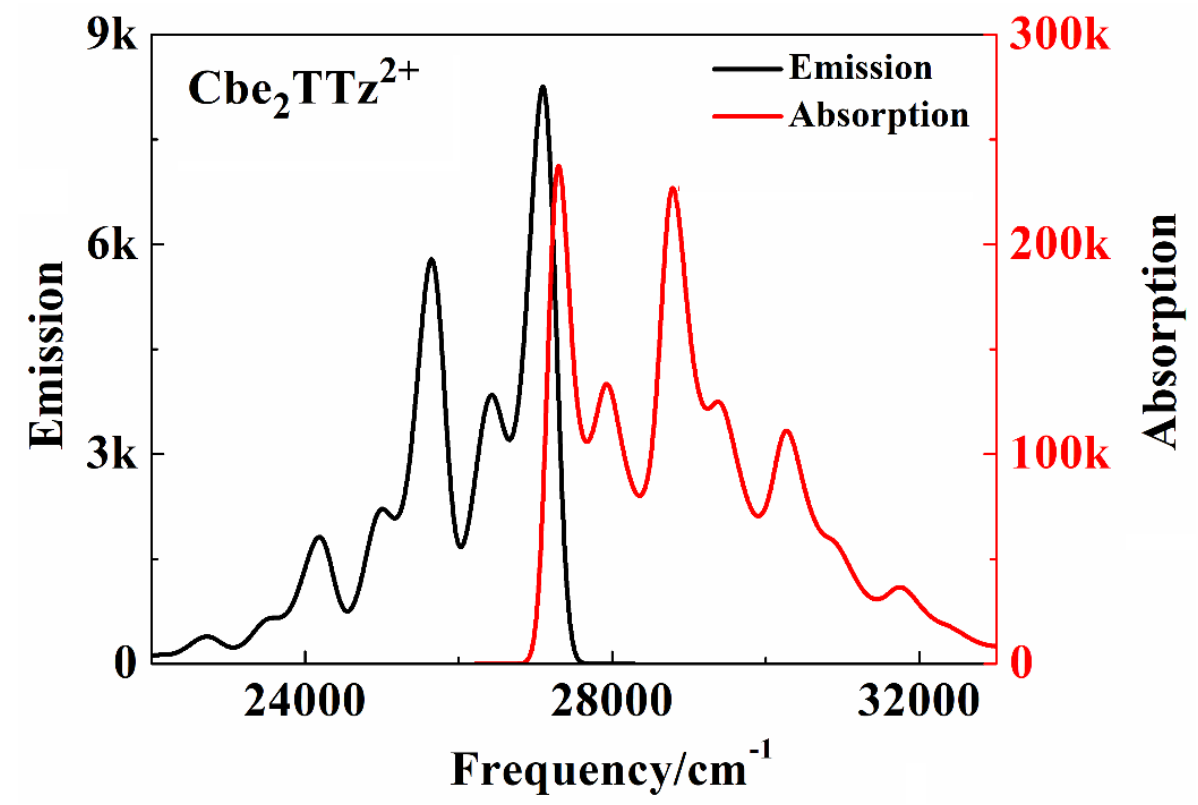

Figure S19. Vibrationally-resolved absorption and emission spectra of $\mathrm{Cbe}_{2} \mathrm{TTz}^{2+}$ at the PBE1PBE/6-31+G(d,p) level. 


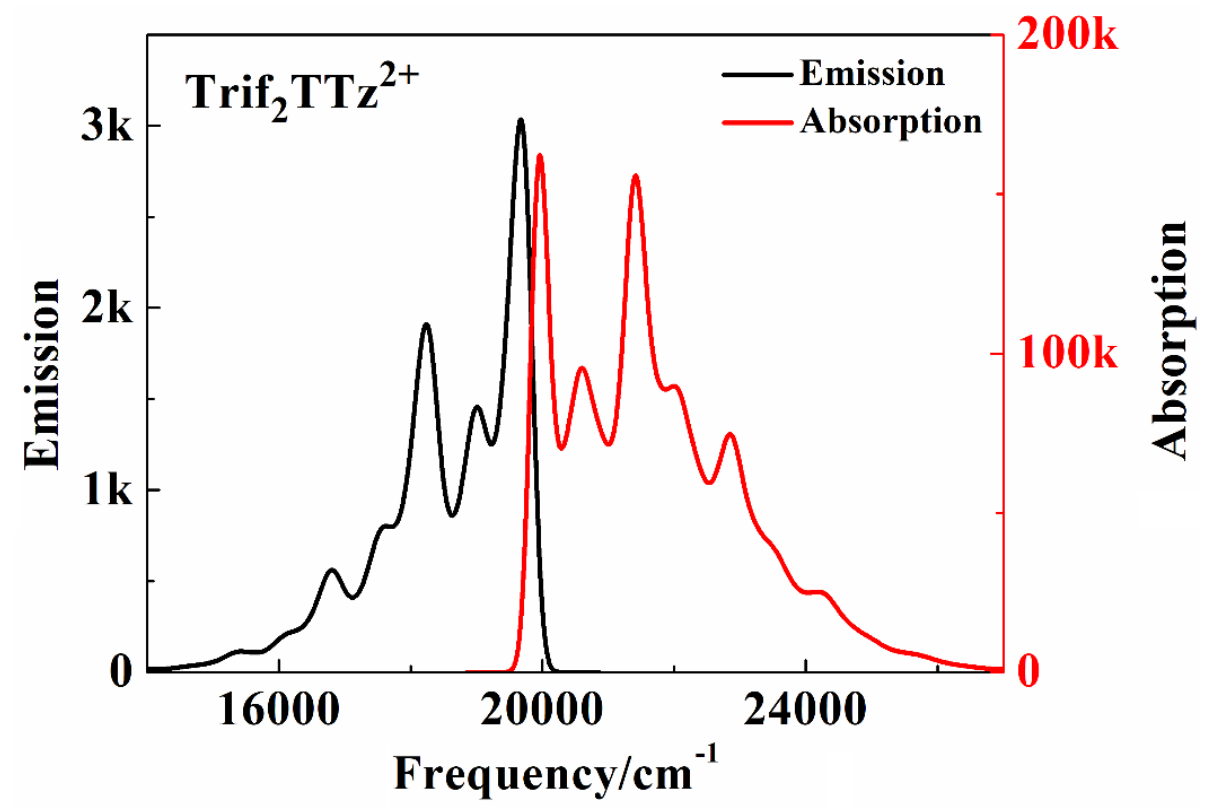

Figure S20. Vibrationally-resolved absorption and emission spectra of $\mathrm{Trif}_{2} \mathrm{TTz}^{2+}$ at the PBE1PBE/6-31+G(d,p) level.

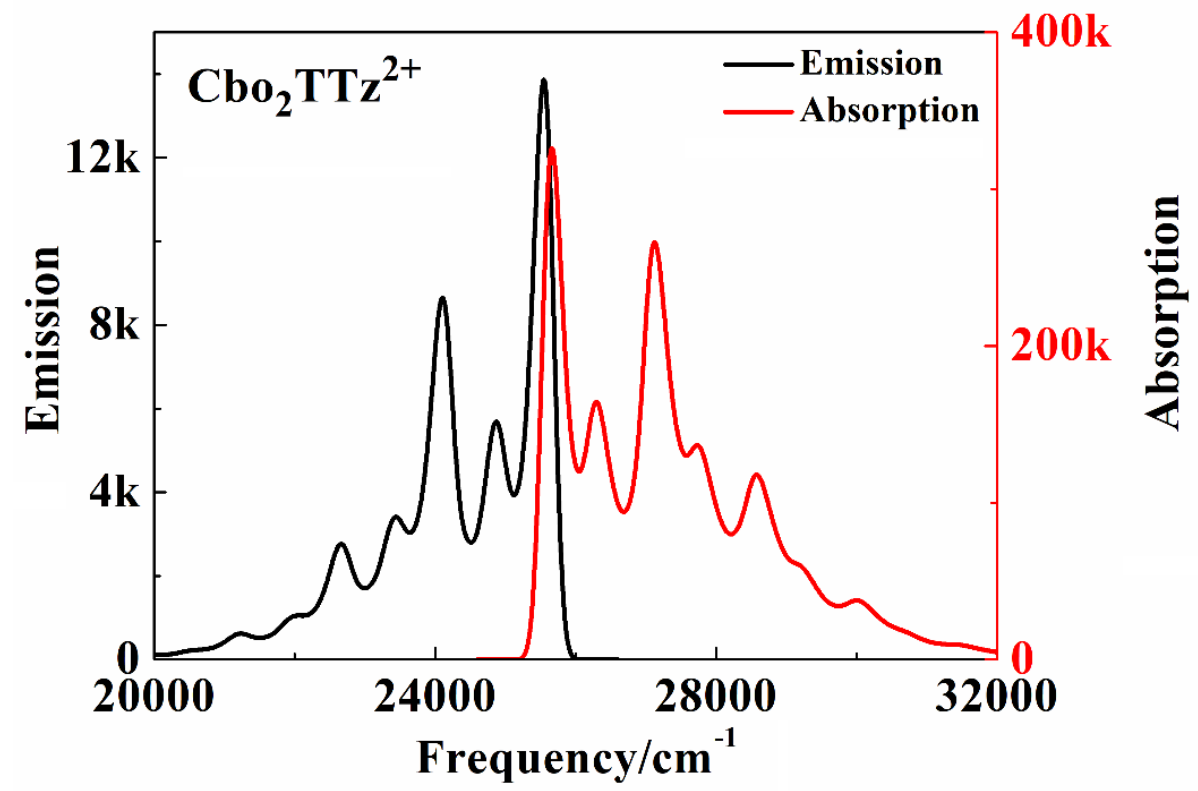

Figure S21. Vibrationally-resolved absorption and emission spectra of $\mathrm{Cbo}_{2} \mathrm{TTz}^{2+}$ at the PBE1PBE/6-31+G(d,p) level. 


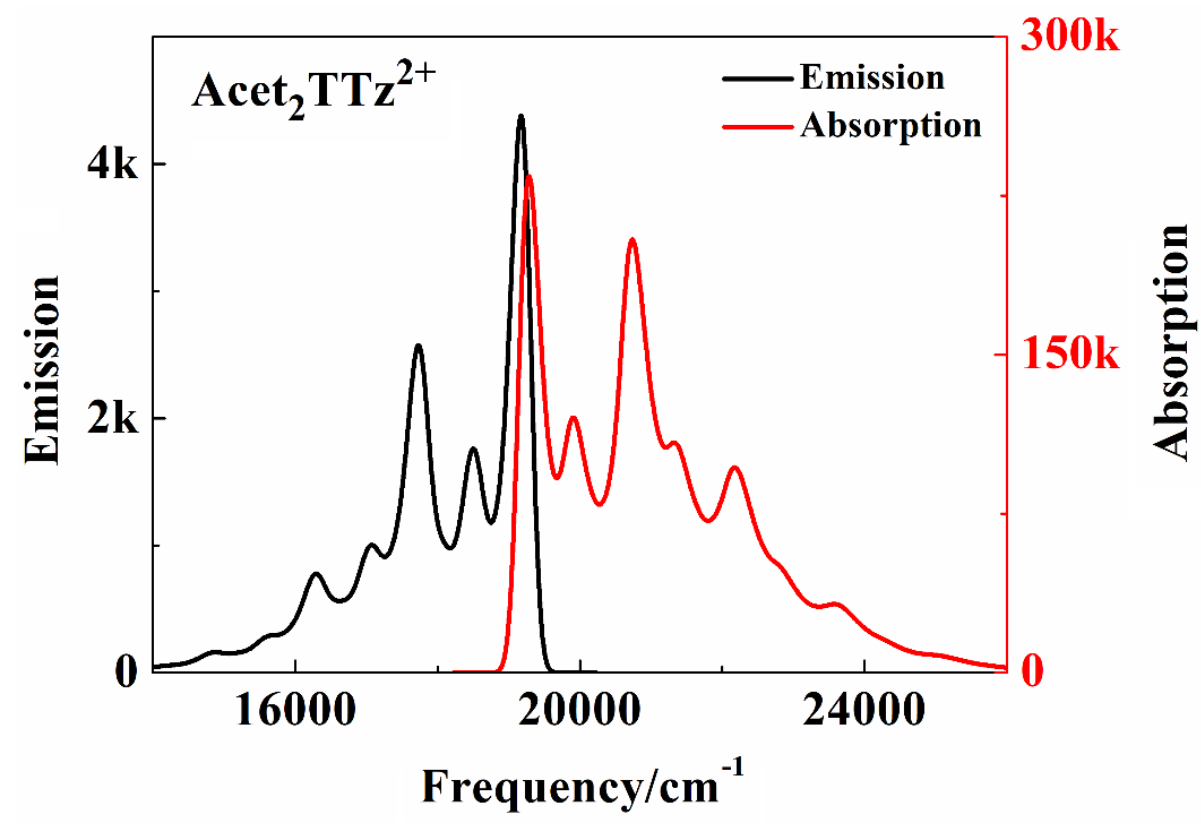

Figure S22. Vibrationally-resolved absorption and emission spectra of $\mathrm{Acet}_{2} \mathrm{TTz}^{2+}$ at the PBE1PBE/6-31+G(d,p) level.

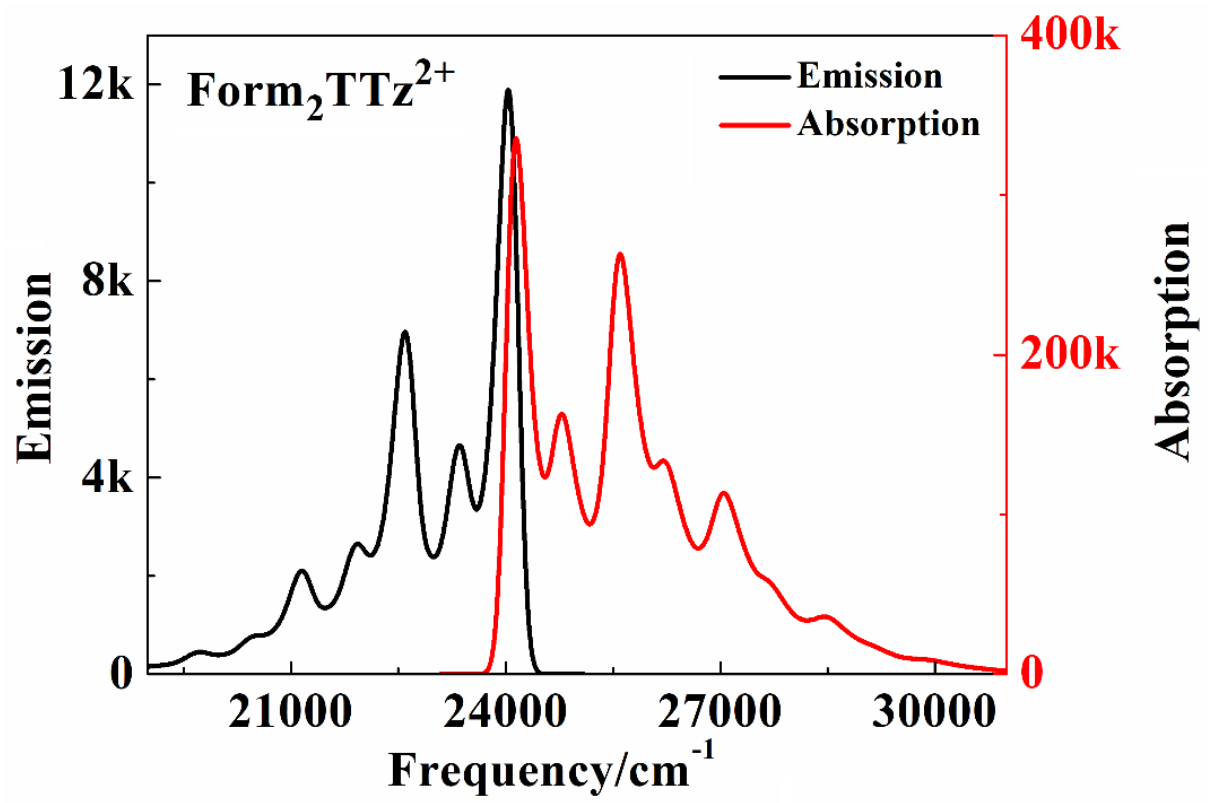

Figure S23. Vibrationally-resolved absorption and emission spectra of Form $_{2} \mathrm{TTz}^{2+}$ at the PBE1PBE/6-31+G(d,p) level. 


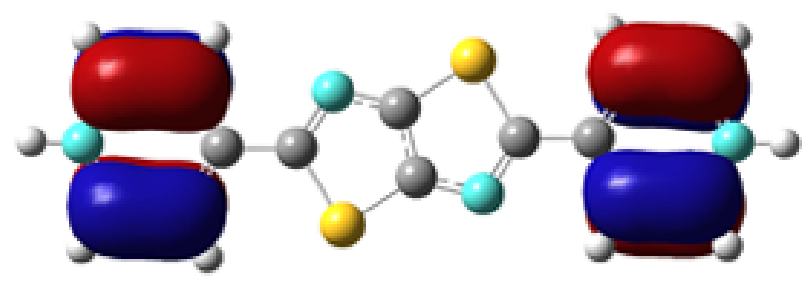

\section{HOMO-2(-8.769eV)}

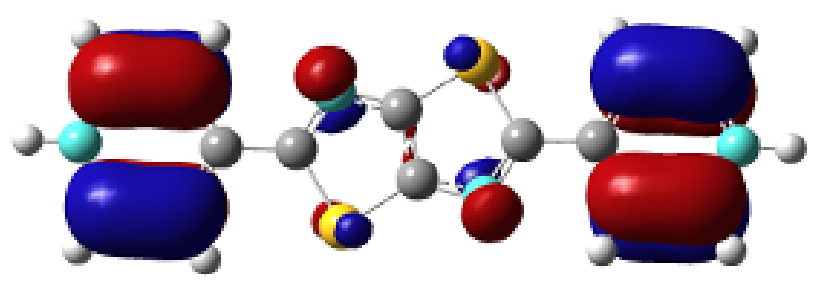

HOMO-3(-8.785eV)

Figure S24. Frontier molecular orbitals of $\mathrm{H}_{2} \mathrm{TTz}^{2+}$ in closed-shell singlet.

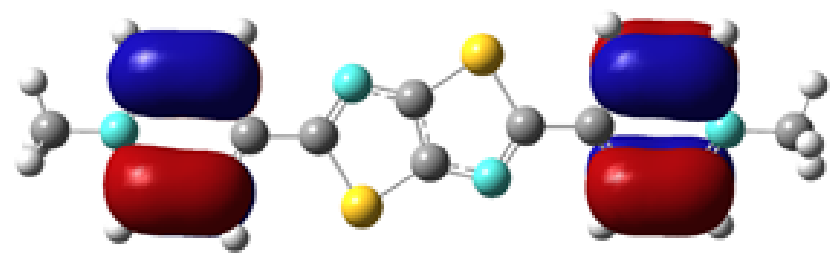

HOMO-2(-8.769eV)

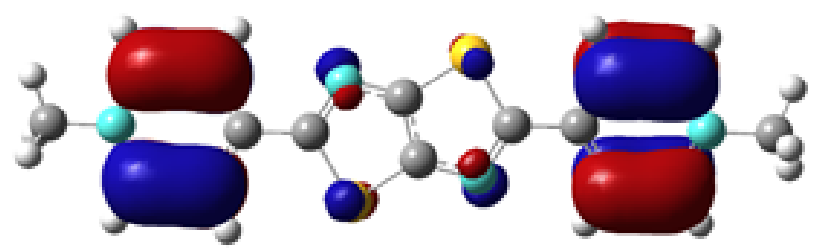

HOMO-3(-8.785eV)

Figure S25. Frontier molecular orbitals of $\mathrm{Me}_{2} \mathrm{TTz}^{2+}$ in closed-shell singlet. 


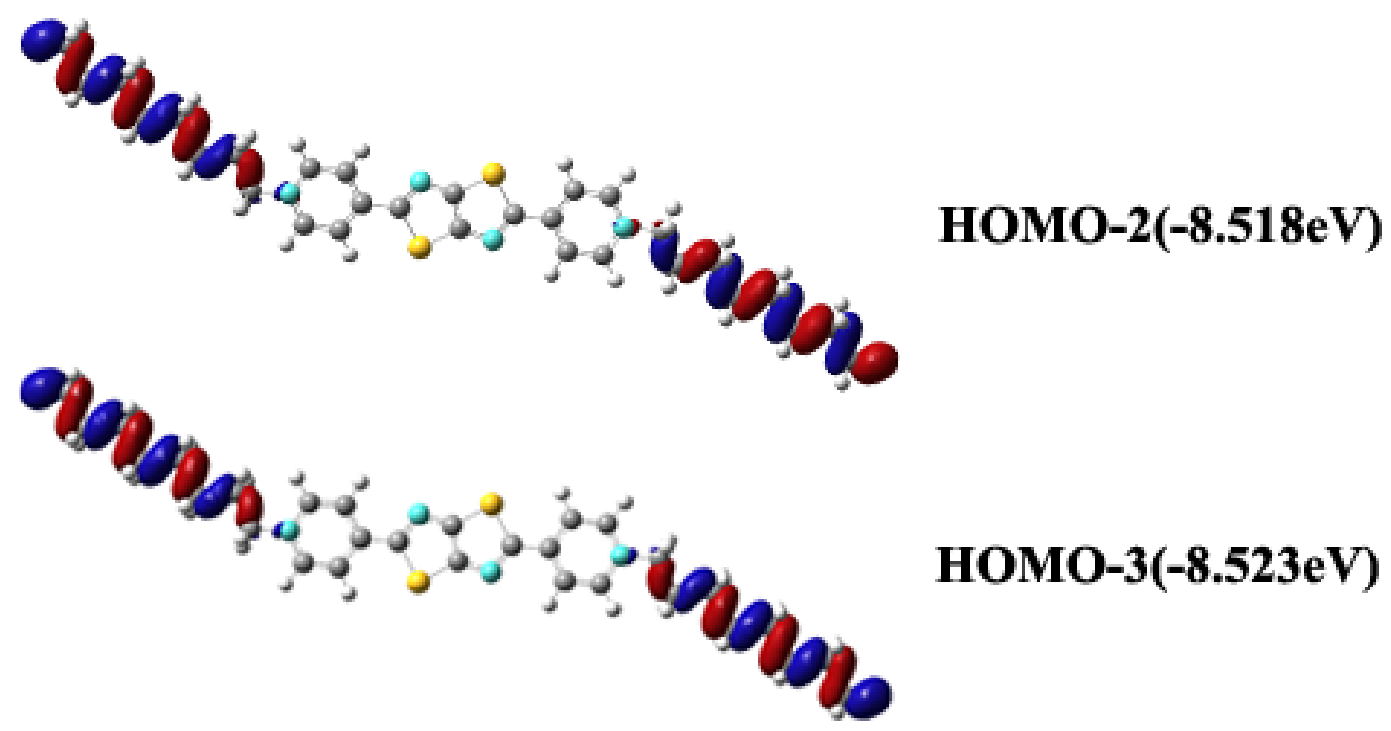

Figure S26. Frontier molecular orbitals of $\mathrm{Oct}_{2} \mathrm{TTz}^{2+}$ in closed-shell singlet.

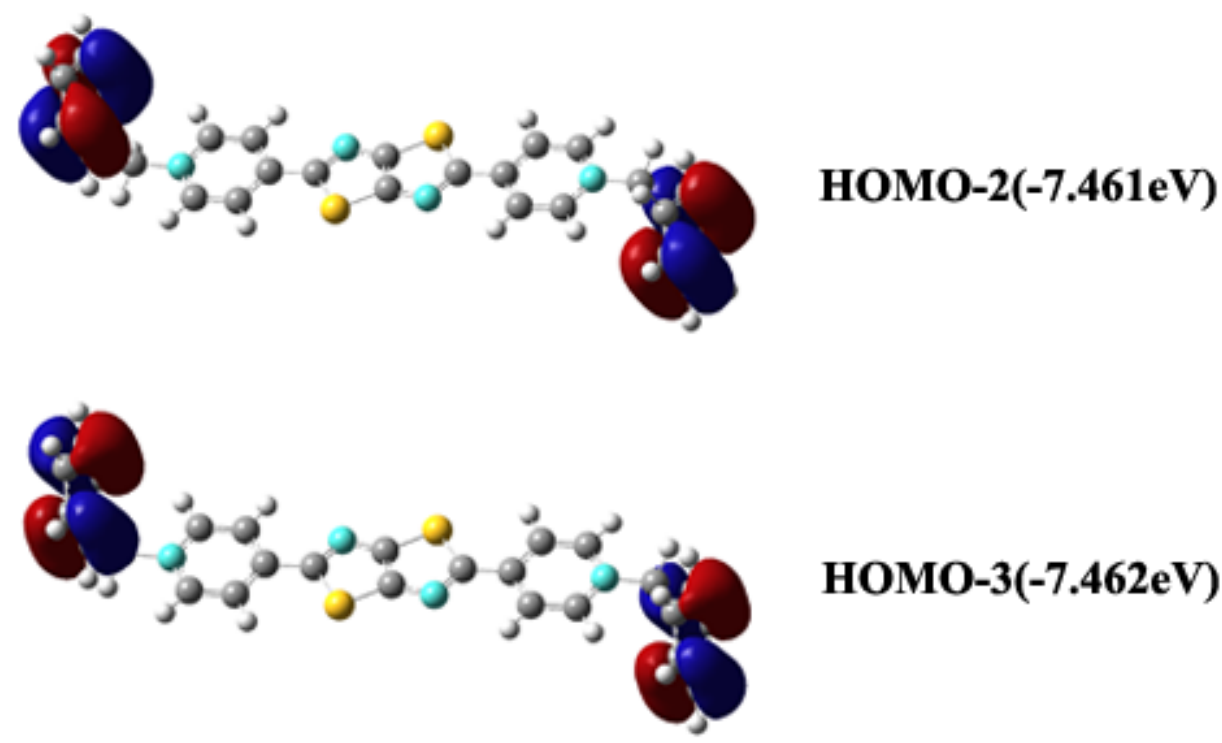

Figure S27. Frontier molecular orbitals of $\mathrm{Bz}_{2} \mathrm{TTz}^{2+}$ in closed-shell singlet. 

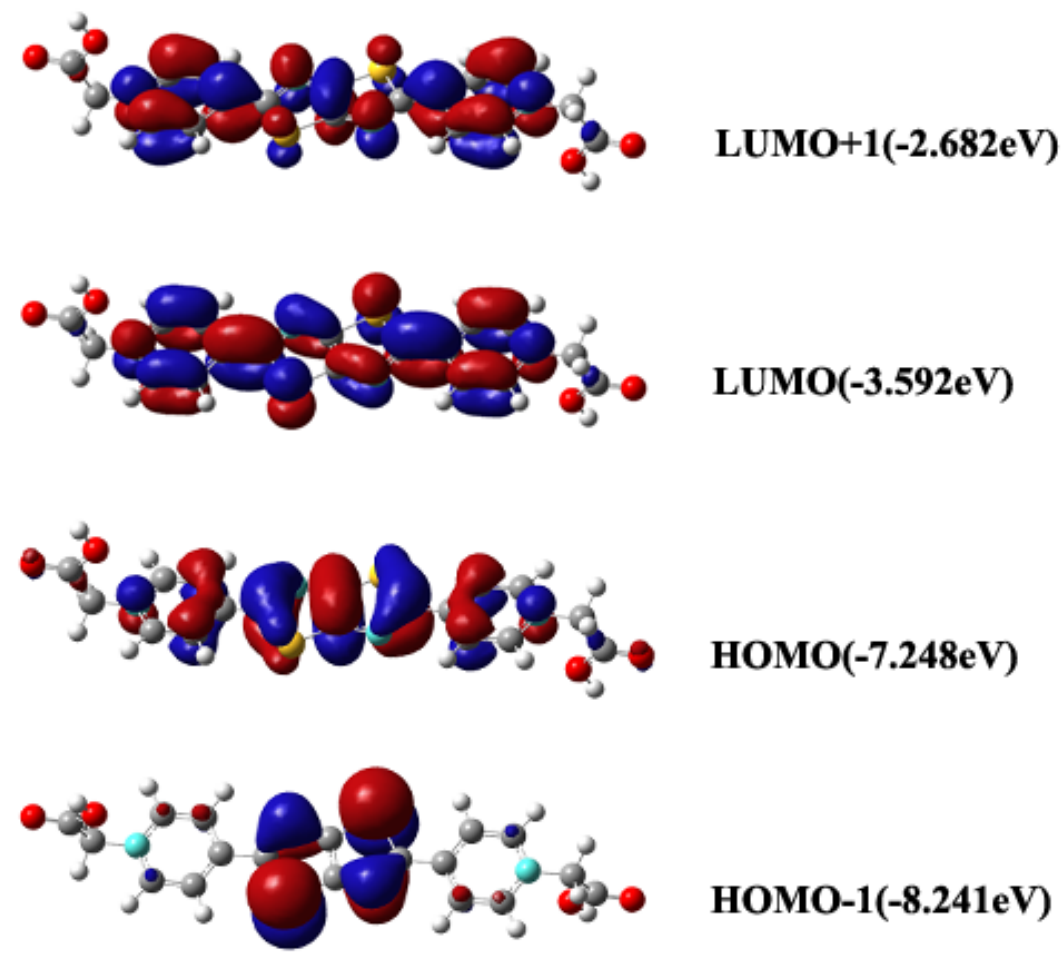

Figure S28. Frontier molecular orbitals of $\mathrm{Met}_{2} \mathrm{TTz}^{2+}$ in closed-shell singlet.
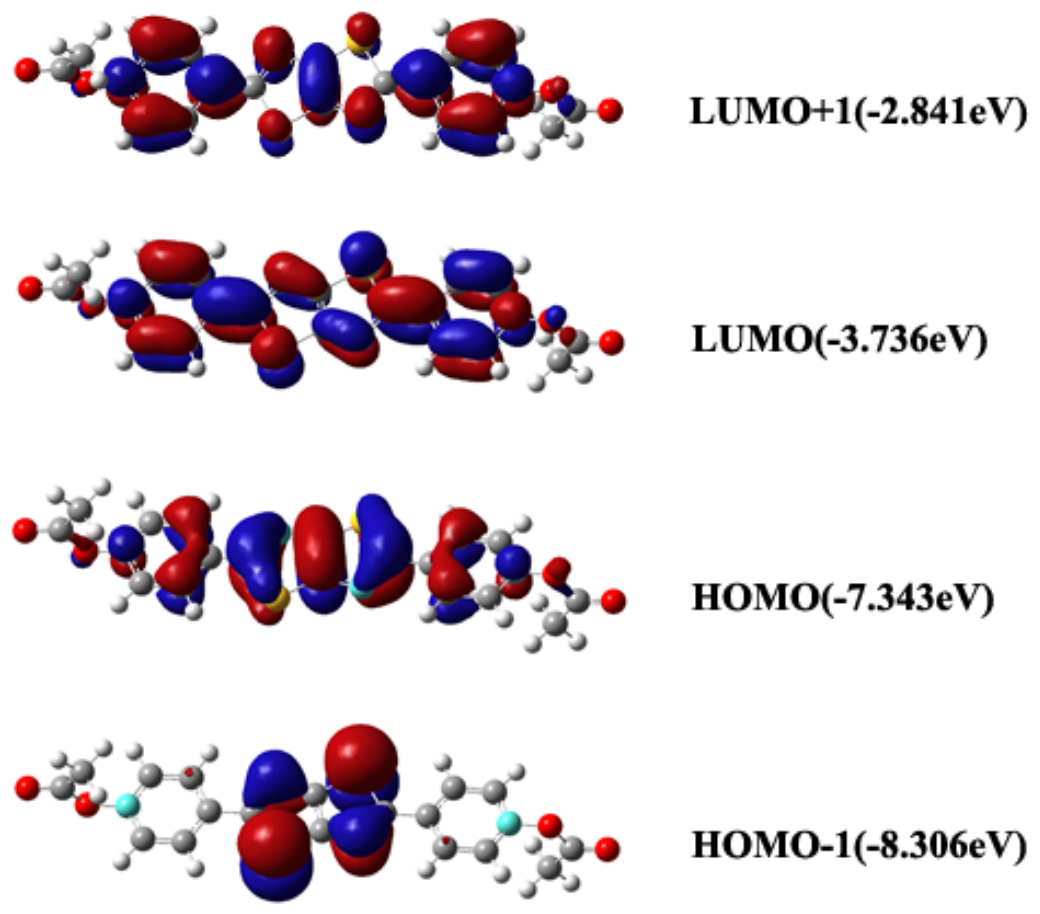

Figure S29. Frontier molecular orbitals of $\mathrm{Cbe}_{2} \mathrm{TTz}^{2+}$ in closed-shell singlet. 

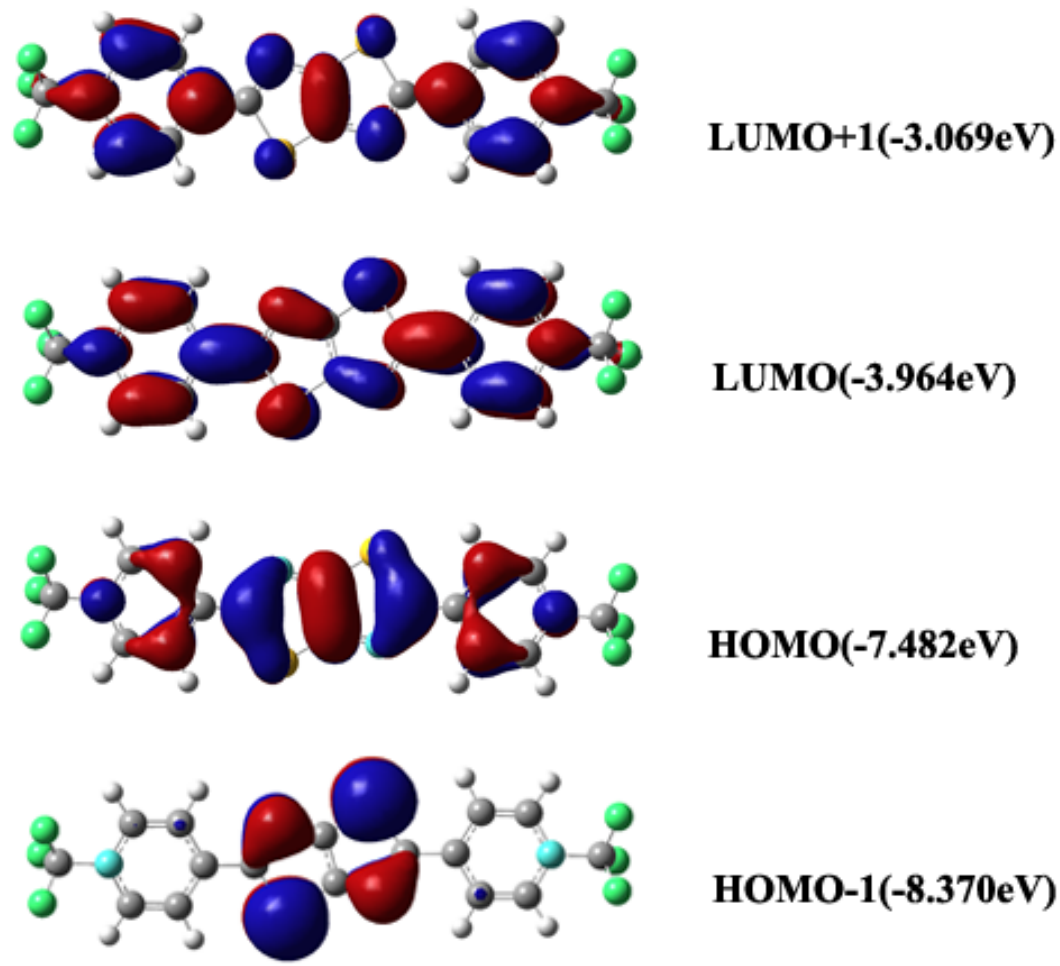

Figure S30. Frontier molecular orbitals of $\operatorname{Trif}_{2} \mathrm{TTz}^{2+}$ in closed-shell singlet.

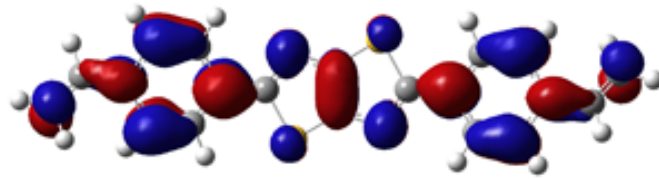

LUMO+1(-2.814eV)

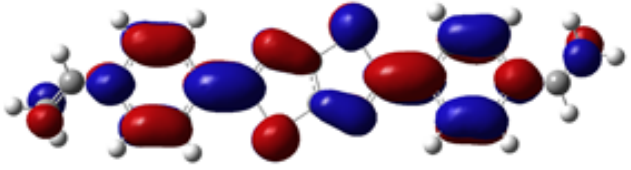

LUMO(-3.651eV)

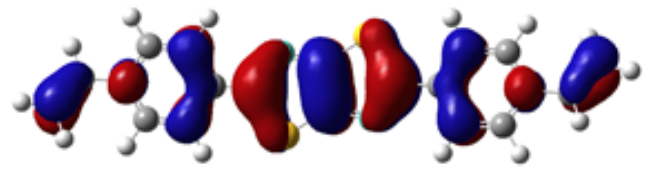

HOMO(-7.126eV)

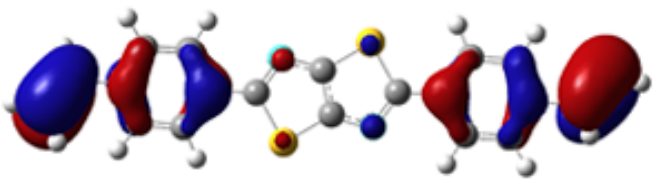

HOMO-1(-8.144eV)

Figure S31. Frontier molecular orbitals of $\mathrm{Eth}_{2} \mathrm{TTz}^{2+}$ in closed-shell singlet. 


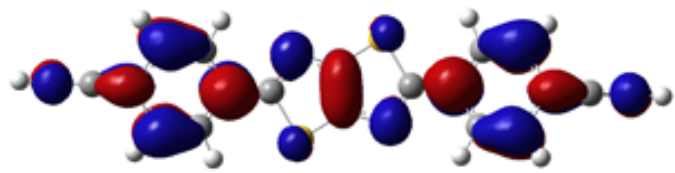

LUMO+1(-3.075eV)

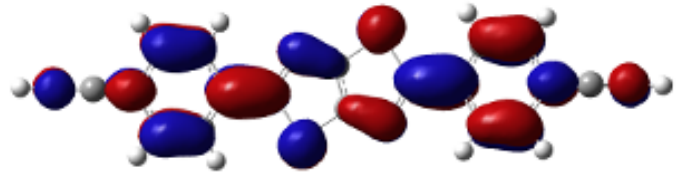

LUMO(-3.920eV)

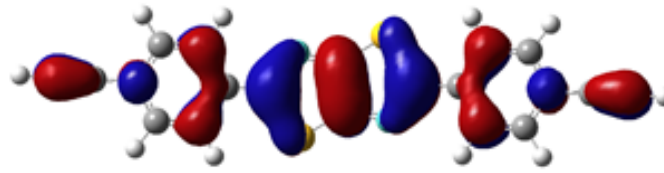

HOMO(-7.283eV)

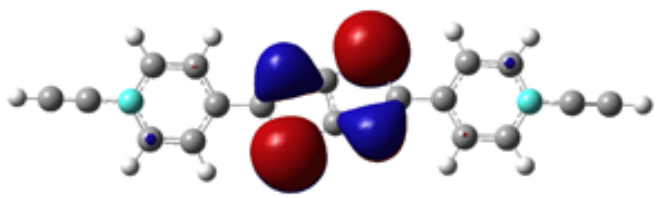

HOMO-1(-8.312eV)

Figure S32. Frontier molecular orbitals of $\mathrm{Ace}_{2} \mathrm{TTz}^{2+}$ in closed-shell singlet. 